Sādhanā, Vol. 19, Part 3, June 1994, pp. 361-399. (C) Printed in India.

\title{
Detailed study of complex flow fields of aerodynamical configurations by using numerical methods
}

\author{
A DAS \\ Department of Numerical Aerodynamics, Institute SM-EA, DLR, \\ Braunschweig, Germany
}

\begin{abstract}
The mathematical physics of fluid flow in a compressible medium, leads to nonlinear partial differential equations or their equivalent integral versions. For the solution of these equations one has generally to resort to numerical methods using mostly finite difference or finite volume schemes, which are well established now. These field methods are very suitable for studying the physical features of complex flows. The present paper gives at first a short sketch of the numerical procedure and thereafter goes into the detailed analysis of the flow fields of delta wings, double-delta wings, delta shaped wing-canard combinations and space vehicles. Further examples include long span wings and wing-bodies at supercritical onflows, flows around propellers and rotors and finally some unsteady flows. The examples cited are selected topics from the extensive studies undertaken in the department of numerical aerodynamics of the DLR in Braunschweig in the course of the last few years.
\end{abstract}

Keywords. Complex flow fields; aerodynamical configurations; numerical aerodynamics,

\section{Introduction}

For the study of complex flows in compressible media it is essential to work with the field solutions of Navier-Stokes equations, or else with the Euler equations when the effects of viscosity and heat conductivity are small, especially at high Reynolds number of the flow. In both cases one has to solve nonlinear partial differential equations, generally by resorting to numerical methods. These methods are well established now and are widely implemented, mostly by using finite-difference or finite-volume schemes. The efficiency of a numerical method is measured by three criteria-good accuracy with robustness, acceptable computing time and easy applicability to complex flow fields. Since the numerical methods for solving nonlinear partial differential equations (Courant et al 1928, 1952; Lax \& Wendroff 1966) have been established, some basic schemes for solving the Euler equations were introduced thereafter (Beam \& Warming 1976; Steger \& Warming 1979; Pulliam \& Steger 1980; Jameson et al 1981; Roe 1981; Whitfield \& Janus 1984; Van Leer 1985; Eberle 1987), and numerical schemes for solving Reynolds-averaged Navier-Stokes equations have 
also been developed (Beam \& Warming 197.8; MacCormack 1982, 1985; Jameson 1985; Pan \& Lomax 1986; Van Leer et al 1987). These basic methods needed however considerable efforts for improved efficency with respect to the three criteria as stated above and the contributions on this line in recent years have been very significant (some selected papers being Radespiel \& Kroll 1985; Kroll \& Jain 1987; Rossow 1987, 1989, 1991; Kroll et al 1989, 1991; Kroll \& Rossow 1990; Radespiel \& Kroll 1990; Radespiel et al 1990; Blazek et al 1991; Swanson \& Radespiel 1991; Blazek 1992). For solving the Reynolds-averaged Navier-Stokes equations for turbulent flows it is essential to model the turbulent exchanges producing viscous stresses. A unified and simple approach is the classical eddy-viscosity modelling, which is now being put in an extended version as outlined (Baldwin \& Lomax 1978; Degani \& Schiff 1983; Johnson \& King 1984). One prerequisite for improved efficiency of numerical schemes is the generation of surface and field grids of outstanding quality, as regards smoothness, orthogonality and proper grid fineness especially in regions of high flow gradients. The technique of grid generation is also well established and is applied for producing body fitted grids of various topologies. The basic methods of grid generation and their implementations are discussed (Eriksson 1982; Thompson et al 1985; Schwarz 1986; Sonar \& Radespiel 1986; Radespiel 1988; Sonar 1989; Findling \& Herrmann 1991; Pahlke \& Kroll 1991; Rossow \& Ronzheimer 1991).

The numerical solutions of Euler and Navier-Stokes equations yield abundant field data in the physical domain around a moving aerodynamical configuration, which can be used for analysing the main features of the flow, both over the boundary surface and in the surrounding space. Such a study is of great importance for complex flow fields involving vortical flows, shock waves and shock boundary layer interactions.

It is well known that slender delta wings moving with moderate to high angles of incidence produce spiralling vortical flows over their upper surface thus causing an appreciable additional lift force, which is termed vortex-lift. This leads to improved aerodynamic properties of the delta-wing aircraft. The flow in the spiralling vortices is complex, being characterized by two special features - loss of total pressure in the spiralling flows and the breakdown of the vortices at high angles of incidence. For some years extensive efforts have been made to study the physics of the vortical flows and to find means for stabilizing them to still higher angles of incidence. Some selected contributions on these lines are cited in the literature (Eriksson \& Rizzi 1983; Murman \& Rizzi 1986; Rizetta \& Shang 1986; Newsome \& Kandil 1987; Kumar \& Das 1988; Longo 1988, 1992; Raj et al 1988; Scherr \& Das 1988; Krause \& Liu 1989; Agrawal et al 1990; Hilgenstock 1990; Hilgenstock \& Vollmers 1990; Longo \& Das 1990; Das 1991; Das \& Longo 1994).

In case of space vehicles using round-nosed wing-body configuration one has to deal with complex flows at subsonic to hypersonic speed regimes involving vortical flows at low speeds and multi-shock flow fields in supersonic and hypersonic velocities. Some interesting studies on these configuration have been made (Risk \& Chaussee 1981; Chaussee et al 1984; Pfitzner \& Weiland 1987; Schöne et al 1990, 1991; Radespiel \& Swanson 1991; Schöne \& Bidault 1991).

Modern transport aircrafts operating with transonic cruising speeds possess long span wings with supercritical aerofoils, thus producing supersonic zones on the upper surface with shock waves and shock induced boundary layer separation. Besides these the wings of a complete aircraft experience interference effects of the fuselage and jet engine nacelles. Again the field solutions using Navier-Stokes and Euler equations are 
well suited to study these effects (Chaussee 1986; Deese \& Agarwal 1987; Volpe \& Jameson 1988; Radespiel 1989; Rossow \& Ronzheimer 1991; Rudmik 1991; Longo 1992; Rossow et al 1992; Wichmann 1992).

In order to optimize the shapes of propeller blades, propfans and helicopter rotors both in regard to aerodynamics and aeroacoustics it is essential to analyse their flow fields and evaluate the load distributions quite in detail. The outer parts of the blades operating at transonic speeds need to have supercritical aerofoil-shapes, as they are involved in producing supersonic zones and shock waves. Recent investigations (Bober et al 1983; Deese \& Agarwal 1988; Kroll 1989) report the advancements in numerical aerodynamics.

Further topics of numerical study are the unsteady flow fields as are involved due to oscillating or plunging wings and due to helicopter rotors in forward motion, producing supercritical flow conditions with oscillating shock waves on the upper surface. Some preliminary studies using numerical solutions of Euler equations are undertaken (Whitfield et al 1987; Nixon 1989; Carstens 1990; Lin \& Pahlke 1991).

In order to validate the results of the numerical solutions of the Euler and Navier-Stokes equations it is necessary to have enough experimental data. With this aim extensive wind-tunnel measurements have been carried out in recent years as are reported (Maynard \& Murphy 1950; Caradonna \& Phillipe 1976; Bornemann \& Surber 1978; Schmitt \& Charpin 1979; Caradonna \& Tung 1981; Brennenstuhl \& Hummel 1982; Lambourne 1982; Redeker et al 1987; Drougge 1988; Hummel 1988; Esch 1989; Radespiel \& Quast 1989; Bergmann et al 1990; Elsenaar \& Hoijemakers 1990; Oelkar 1990; Goodard et al 1991).

In the present paper some essential féatures of complex flows around wings, wing-bodies, propellers and rotors as obtained from numerical solutions of Eulerand Navier-Stokes equations are illustrated. The underlying physics helps to understand many of the findings already known from experimental investigations.

\section{Basic equations of flow fields and their numerical simulation and solution}

The disturbance fields arising from the motion of wings and bodies in a compressible viscous medium are adequately described by the Navier-Stokes equations, being based on the conservation laws of mass, momentum and energy in an elementary volume moving with the coordinate system. If the flow is concerned with turbulent exchanges of momentum, a major task is to model these viscous stresses in order to have a complete formulation of the mathematical physics. The usual procedure is to use the classical eddy-viscosity modelling or an equivalent kinematic-dynamical relation.

\subsection{The field equations in viscous and nonviscous medium}

For a fluid medium at standard pressure and temperature having negligible body- or external field-forces the physics of the flow can be fully described by the NavierStokes equations in the following form:

$$
\frac{D \rho}{D t}+\rho \operatorname{div} \bar{V}=0
$$




$$
\begin{aligned}
& \frac{D(\rho \mathbf{V})}{D t}+(\rho \mathbf{V}) \operatorname{div} \mathbf{V}+\operatorname{grad} p-\left\{\operatorname{div} \sigma_{v}\right\}=0, \\
& \frac{D(\rho E)}{D t}+(\rho E) \operatorname{div} \mathbf{V}+\operatorname{div}(p \mathbf{V})-\left\{\operatorname{div}(k \operatorname{grad} T)+\operatorname{div}\left(\mathbf{V} \cdot \boldsymbol{\sigma}_{v}\right)\right\}=0,
\end{aligned}
$$

where

$$
\begin{array}{ll}
\frac{D}{D t}=\frac{\partial}{\partial t}+\mathrm{V} \cdot \mathrm{grad} ; & E=e+\left(V^{2} / 2\right), \\
\mathrm{V}=i u+j v+k w ; & \sigma_{v} \equiv \text { stress tensor. }
\end{array}
$$

The set of equations forming (1) contains the unknowns $u, v, w, p, \rho, T$ and the viscous stress tensor $\sigma_{v}$, thus having more unknowns than the number of equations. An auxiliary relation can be made use of by including the equation of state, which reads

$$
p=\rho R T=(\kappa-1) \rho\left[E-\left(u^{2}+v^{2}+w^{2}\right) / 2\right] .
$$

While the components of the stress tensor $\sigma_{v l}$ for laminar flows are easily modelled when the molecular coefficient of viscosity $\mu_{m}$ is known, the corresponding stress tensors $\sigma_{v}$ for turbulent flows can be based on the Reynolds averaged turbulent stresses expressed as:

$$
\begin{aligned}
& \left.\left.\sigma_{i i}\right|_{t} \equiv \tau_{i i}\right|_{t}=-\rho \overline{u_{i}^{\prime 2}} \ll p, \text { (normal stress) } \\
& \left.\sigma_{i j}\right|_{t}=\left.\tau_{i j}\right|_{t}=-\rho \overline{u_{i}^{\prime} u_{j}^{\prime}}, \quad \text { (tangential stress) }
\end{aligned}
$$

where $u_{i}^{\prime}, u_{j}^{\prime}$ etc. are the turbulent fluctuations of the velocities $u, v, w$.

Using the concept of eddy-viscosity these two stress-terms can be written in general as

with

$$
\overline{\rho u_{i}^{\prime} u_{j}^{\prime}}=\mu_{i}\left[\frac{\partial u_{i}}{\partial x_{j}}+\frac{\partial u_{j}}{\partial x_{i}}\right]-\frac{2}{3} \mu_{i} \frac{\partial u_{k}}{\partial x_{k}} \delta_{i j}
$$

$$
\mu_{t}=\rho \overline{l v}=\rho \bar{l}^{2}|\tilde{\omega}|
$$

where $\bar{l}$ denotes the length scale and $\bar{v}$ the velocity scale of the turbulent fluctuations, while $|\tilde{\omega}|=\left(\gamma_{i}^{2}+\gamma_{j}^{2}+\gamma_{k}^{2}\right)^{1 / 2}$ is the vorticity in the viscous region. Thus (4) has the same expression as for laminar flows with $\mu_{m}$ replaced by $\mu_{t}$, which can be determined by using the Baldwin-Lomax (1978) model. The molecular viscosity $\mu_{m}$ is yielded by the classical Sutherland formula.

The coefficient of heat conduction $k$ in the (1) is given by

$$
k=\left\{\left(\mu_{m} / \operatorname{Pr}_{l}\right)+\left(\mu_{t} / \operatorname{Pr}_{t}\right)\right\} \bar{c}_{p},
$$

where $\operatorname{Pr}_{l}$ and $\operatorname{Pr}_{t}$ are the Prandtl numbers in laminar and turbulent flows.

For analysing some details of the flow fields containing spiralling flows and vortices it is often convenient to work with the momentum equation in Lambs' version, which reads

$$
\frac{D V}{D t}=\frac{\partial V}{\partial t}+\operatorname{grad} \frac{V^{2}}{2}+\gamma \times V=-\frac{\operatorname{grad} p}{\rho}+\frac{\operatorname{div} \sigma_{v}}{\rho}
$$


where $\sigma=-p I+\sigma_{v}$ denotes the stress dyad, with $\sigma_{v}$ as viscous stress tensor. Using the second law of thermodynamics (6) can be rewritten in the Lamb-Crocco version yielding

$$
\begin{aligned}
& (\partial V / \partial t)+\operatorname{grad}\left(V^{2} / 2\right)+\gamma \times \mathbf{V}=T \operatorname{grad} s-\operatorname{grad} h+\left(\operatorname{div} \sigma_{v} / \rho\right), \\
& (\partial V / \partial t)+\gamma \times \mathbf{V}=-\operatorname{grad} h_{0}+T \operatorname{grad} s+\left(\operatorname{div} \sigma_{v} / \rho\right) .
\end{aligned}
$$

Furthermore, it may be necessary to use cylindrical coordinates for evaluating the terms of (6) to (8), thus defining

$$
\begin{aligned}
\operatorname{grad} & =i \frac{\partial}{\partial r}+j \frac{l}{r} \frac{\partial}{\partial \vartheta}+k \frac{\partial}{\partial x} \\
\operatorname{div} \sigma_{v} & =\frac{l}{r}\left\{\frac{\partial\left(\sigma_{v r} \cdot r\right)}{\partial r}+\frac{\partial\left(\sigma_{v g} \cdot r\right)}{\partial \vartheta}+\frac{\partial\left(\sigma_{v x} \cdot r\right)}{\partial x}\right\}
\end{aligned}
$$

with $\gamma=i \gamma_{r}+j \gamma_{9}+k \gamma_{x}$ and $\mathrm{V}=i v_{r}+j v_{9}+k v_{x}$.

\subsection{The Euler equations of a flow field in a perfect medium}

For flows in a compressible medium with vanishingly small effects of viscosity and heat conductivity, the terms in the brackets of (1) can be neglected. Using the relation of the first equation in the other two (1) can be expressed in a reduced version, which is commonly known as the classical set of Euler equations for a flow in a. perfect medium. They read

$$
\begin{gathered}
\frac{D \rho}{D t}+\rho \operatorname{div} \mathbf{V}=0, \\
\rho \frac{D V}{D t}+\operatorname{grad} p=0, \\
\rho \frac{D E}{D t}+\operatorname{div}(p \mathbf{V})=0,
\end{gathered}
$$

with $p=(\kappa-1) \rho e=\rho R T$, thus having six equations for the six unknowns $\rho, u, v, w, p$ and $T$.

The field equations described in this section are well suited to study complex flow fields as depicted in figure 1 and are applicable for all flow regimes from subsonic to hypersonic velocities.

\subsection{The generation of field grids around aerodynamical configurations}

The numerical field methods using finite difference or finite volume formulations need suitable field grids around the moving body - the grid spacings should conform to the physical requirements of good flow resolution in regions where high flow gradients are expected and must enable the capture of flow details in the viscous layer close to the body surface. In contrast to this, the grids near the far field boundary can be sparse by having wider stretchings. The quality of the field grids is measured by the smoothness and primarily by the orthogonality to each other and also to the frictional 


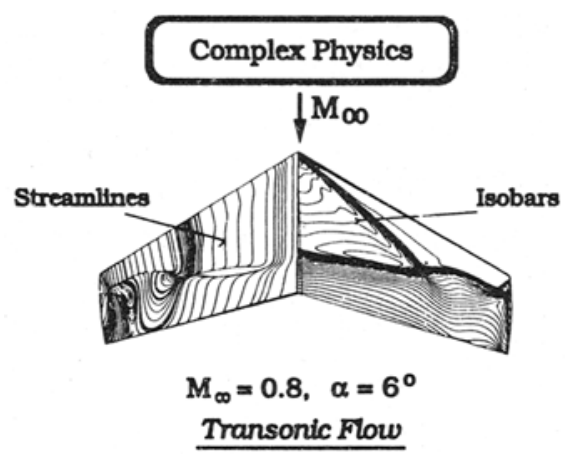

\section{Complex Configurations}

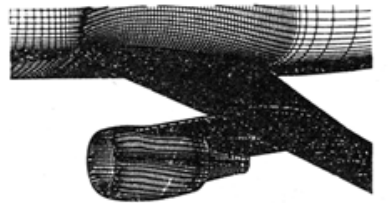

Transport Aircraft

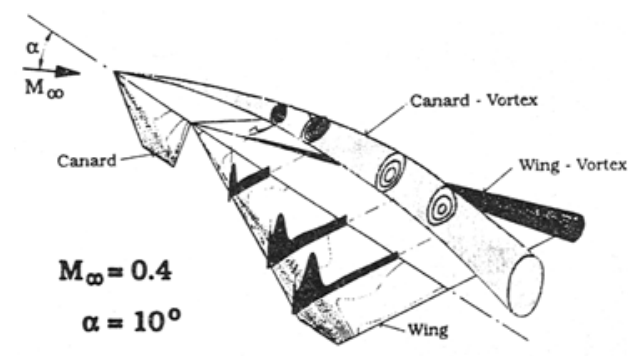

Vortical Flows

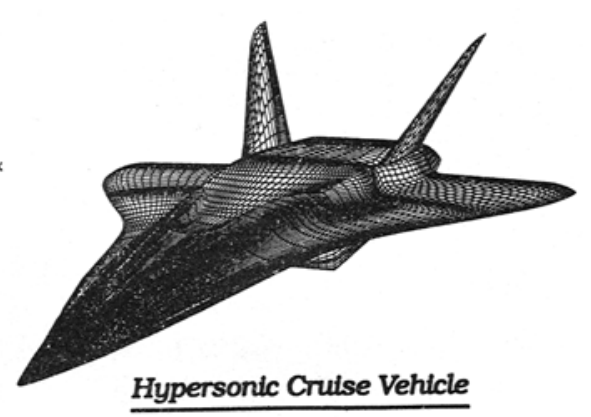

Figure 1. Some complex flow fields in subsonic to hypersonic speed regimes.

surface. The grid topologies to be used depend usually on the nature of the body geometry - they being commonly classified under the notations $\mathrm{O}$-, $\mathrm{C}$-, and $\mathrm{H}$-grids for a given plane, thus leading to the combinations $\mathrm{O}-\mathrm{O}, \mathrm{C}-\mathrm{O}, \mathrm{C}-\mathrm{H}$ etc. for two orthogonal planes, as are shown in figure 2 . The grid generation follows three essential steps comprising the following.

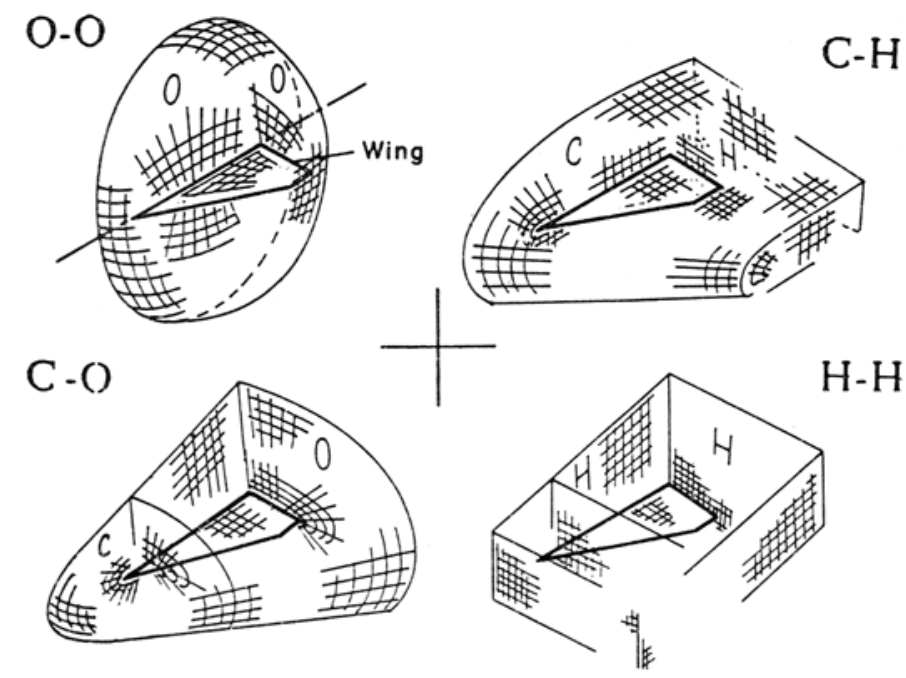

Figure 2. Standard grid topologies for numerical solution of the field equations of bodies moving in an unbounded medium. 
- Analytical description of the surface geometry of the moving body, by using interpolation techniques based on cubic splines or Bezier-polynomials. A very elegant procedure is the method described as Coon's surface using linear interpolation with specified pairs of opposite edge points and the corner points of the surface boundary. This scheme is based on Lagrange polynomials.

- Generation of surface grids comprising the distribution of grid points of the solid body, the far field and on the inner cuts introduced in the field. It is essential to have grid clusterings in regions, where high flow gradients are expected. This is realized by using suitable stretching functions.

- Generation of field grids between the solid surface and the far field, for which one of the two basic methods can be followed, both being quite well established and widely implemented till now. These are:

a) Initial layout of body-fitted curvilinear coordinates based on algebraic transfinite interpolation schemes with subsequent refinement of the grids for smoothness and orthogonality. For achieving this, the spacings in physical- and computationaldomains are interrelated by the solution of elliptic system of equations with source terms, being expressed as Poisson equations $\nabla^{2} X^{i}=P^{i}$, where

$$
\nabla^{2}=\frac{\partial^{2}}{\partial \xi^{2}}+\frac{\partial^{2}}{\partial \eta^{2}}+\frac{\partial^{2}}{\partial \zeta^{2}}
$$

b) Use of numerical schemes based on biharmonic equations having the expression

$$
\text { or } \begin{aligned}
\nabla^{4} X^{i} & =0, \\
\nabla^{2} \cdot \nabla^{2} X^{i} & =0 .
\end{aligned}
$$

Setting $\nabla^{2} X^{i}=P^{i}$, (11) decomposes into two elliptic systems of equations for the whole field

$$
\begin{aligned}
& \nabla^{2} X^{i}=P^{i} \\
& \nabla^{2} P^{i}=0 .
\end{aligned}
$$

In both the methods for generating field grids one is to prescribe the first grid spacings normal to the solid surface, which will then yield the $P^{i}$-values at the inner boundary. While in method (b) the $P^{i}$-values in the whole field are yielded by the solution of the Laplace equation $\nabla^{2} P^{i}=0$, the method (a) needs an interpolation of the source terms $P^{i}$ from the initial data at the solid surface and at the far field, the latter being usually assumed to have zero source strengths.

Generation of surface grids and field grids around a wing-body combination has been depicted in figure 2. Depending on the storage capacity of the computer it is normally necessary to subdivide the grid space in multiblocks and handle the blocks in turn.

\subsection{Numerical simulation of the field equations and the method of solution}

The Navier-Stokes equations describing the physics of flow in a compressible viscous medium, as expressed in (1), can be written in divergence form in the following way:

$$
\frac{\partial \rho}{\partial t}+\operatorname{div}(\rho \mathbf{V})=0
$$




$$
\begin{aligned}
& \frac{\partial(\rho V)}{\partial t}+\operatorname{div}(\mathbf{V} ; \rho \mathbf{V})+\operatorname{div}(p I)-\left\{\operatorname{div} \sigma_{v}\right\}=0, \\
& \frac{\partial(\rho E)}{\partial t}+\operatorname{div}(\rho E \mathbf{V})+\operatorname{div}(p V)-\left\{\operatorname{div}(k \operatorname{grad} T)+\operatorname{div}\left(\mathbf{V} \cdot \boldsymbol{\sigma}_{v}\right)\right\}=0,
\end{aligned}
$$

with $I=(i: i+j: j+k: k)$ as a unit dyad. The divergence of the momentum flux in the second expression contains a dyadic product comprising all the nine components of the flux in the three coordinate directions. For a perfect medium the terms in the curly brackets drop out and (13) reduces then to the Euler equations in divergence form.

For numerical simulation of (13) it is essential to rewrite it in a universal vector form as:

$$
(\partial U / \partial t)+\nabla \cdot \chi=0
$$

where the solution vector $\mathbf{U}$ and the flux tensor $\chi$ are defined as

$$
\mathbf{U}=\left[\begin{array}{c}
\rho \\
\rho u \\
\rho v \\
\rho w \\
\rho E
\end{array}\right], \quad \chi=\left[\begin{array}{l}
\rho \mathbf{V} \\
\rho u \mathbf{V}+p \mathbf{i}_{x}+\sigma_{x x}+\tau_{x y}+\tau_{x z} \\
\rho v \mathbf{V}+p \mathbf{i}_{y}+\sigma_{y y}+\tau_{y x}+\tau_{y z} \\
\rho w \mathbf{V}+p \mathbf{i}_{z}+\sigma_{z z}+\tau_{z x}+\tau_{z y} \\
\rho E \mathbf{V}+p l+\sigma_{v} \cdot \mathbf{V}-k \operatorname{grad} T
\end{array}\right],
$$

with the viscous flux tensor

$$
\sigma_{v} \cdot \mathbf{V}=\left[\begin{array}{ccc}
\sigma_{x x} & \tau_{x y} & \tau_{x z} \\
\tau_{y x} & \sigma_{y y} & \sigma_{y z} \\
\tau_{z x} & \tau_{z y} & \sigma_{z z}
\end{array}\right] \cdot\left[\begin{array}{c}
u \\
v \\
w
\end{array}\right]
$$

In the Cartesian system of coordinates the flux tensor of (15) can be split as:

$$
\chi=F \mathbf{i}_{x}+G \mathbf{i}_{y}+H \mathbf{i}_{z},
$$

so that (14) can be rewritten as

$$
\frac{\partial U}{\partial t}+\frac{\partial E}{\partial x}+\frac{\partial G}{\partial y}+\frac{\partial H}{\partial z}=0
$$

For a finite volume element $\Omega$ enclosed by a surface $S,(14)$ can be expressed in integral form. If the volume integral of the flux tensor is converted to surface integral by using the Gauss-theorem, one obtains:

$$
\frac{\partial \bar{U}}{\partial t}+\frac{1}{\Omega} \int_{S} \chi \cdot \mathrm{nd} S=0
$$

where $\bar{U}=\frac{1}{\Omega} \int_{\Omega} U \mathrm{~d} \Omega$, and $\mathrm{d} S$ is a surface element of $S$ with $\mathbf{n}$ as its unit outer-normal vector.

In case of small effects of viscosity and heat conductivity the corresponding terms 
in (15) can be dropped, thus leading to the integral version of the Euler equations. The Navier-Stokes equations for unsteady flows as expressed in (13) being basically of hyperbolic type need the specification of the following conditions for the solution of a given problem.

(a) The initial condition as prescribed by the onflow at the time $t=t_{0}$.

(b) Boundary condition on the solid surface

pressure: $\quad \partial p / \partial n=0, \quad$ in the viscous layer close to the solid boundary;

velocity: $\quad \mathbf{V}=0, \quad$ no slip at the solid surface;

temperature: $\begin{cases}k(\partial T / \partial n)=0, & \text { adiabatic condition at the wall; } \\ T=T_{w}, & \text { isothermal condition at the wall. }\end{cases}$

(In case of inviscid flows the boundary condition at the solid surface simplifies to $\mathbf{V} \cdot \mathbf{n}=\mathbf{V}_{\infty} \cdot \mathbf{n}+\Delta \mathbf{V} \cdot \mathbf{n}=\mathbf{V}_{s} \cdot \mathbf{n}$, where $\mathbf{V}_{s}$ denotes unsteady motion of the body surface).

(c) Boundary condition at the far field is based on the characteristic relations, so that the propagation of information from inside and outside are properly matched, thus preventing spurious reflections into the enclosed domain.

(d) Condition of periodicity at the inner cuts used in the solution and also of matching of the flow variables at the block boundaries of multiblock grid topologies.

The numerical simulation of the unsteady Navier-Stokes or Euler equations in conservation law form can be undertaken by converting into a finite difference equation, preferably by using body-fitted coordinates.

If curvilinear coordinates are used based on the coordinate transformations

$$
\xi=\xi(x, y, z) ; \quad \eta=\eta(x, y, z) ; \quad \zeta=\zeta(x, y, z),
$$

then the unit vectors and the flux tensors are to be redefined in the $\xi, \eta, \zeta$ coordinates, by using the metric coefficients and the determinant $J$ of the Jacobian matrix.

$$
\begin{aligned}
& \nabla=\mathbf{i}_{\xi} \frac{\partial}{\partial \xi}+\mathbf{i}_{\eta} \frac{\partial}{\partial \eta}+\mathbf{i}_{\zeta} \frac{\partial}{\partial \zeta}, \\
& \tilde{F}=\left[F \frac{\partial \xi}{\partial x}+G \frac{\partial \xi}{\partial y}+H \frac{\partial \xi}{\partial z}\right] \frac{1}{J(\xi, \eta, \zeta)} \\
& \tilde{G}=\left[F \frac{\partial \eta}{\partial x}+G \frac{\partial \eta}{\partial y}+H \frac{\partial \eta}{\partial z}\right] \frac{1}{J(\xi, \eta, \zeta)} \\
& \tilde{H}=\left[F \frac{\partial \zeta}{\partial x}+G \frac{\partial \zeta}{\partial y}+H \frac{\partial \zeta}{\partial z}\right] \frac{1}{J(\xi, \eta, \zeta)}
\end{aligned}
$$

and $\tilde{U}=U / J(\xi, \eta, \zeta)$, while the volume $\tilde{\Omega}=\Omega \cdot J(\xi, \eta, \zeta)$. Hence (17) takes the following form

$$
\frac{\partial \tilde{U}}{\partial t}+\frac{\partial \tilde{F}}{\partial \xi}+\frac{\partial \tilde{G}}{\partial \eta}+\frac{\partial \tilde{H}}{\partial \zeta}=0
$$

thus retaining the conservation law form.

The numerical simulation of (20) in finite-difference form can be undertaken by using explicit or implicit formulations. 
Explicit scheme

$$
U_{i j k}^{(n+1)}=U_{i j k}^{(n)}-\Delta t\left\{\frac{D_{-}}{\Delta \xi} F_{i j k}^{(n)}+\frac{D_{-}}{\Delta \eta} G_{i j k}^{(n)}+\frac{D_{-}}{\Delta \zeta} H_{i j k}^{(n)}\right\} .
$$

Implicit scheme

$$
\begin{aligned}
\tilde{U}_{i j k}^{(n+1)}=U_{i j k}^{(n)}-\frac{\Delta t}{2} & {\left[\left\{\frac{D_{0}}{\Delta \xi} F_{i j k}^{(n)}+\frac{D_{0}}{\Delta \eta} G_{i j k}^{(n)}+\frac{D_{0}}{\Delta \zeta} H_{i j k}^{(n)}\right\}\right.} \\
+ & \left.\left\{\frac{D_{0}}{\Delta \xi} F_{i j k}^{(n+1)}+\frac{D_{0}}{\Delta \eta} G_{i j k}^{(n+1)}+\frac{D_{0}}{\Delta \zeta} H_{i j k}^{(n+1)}\right\}\right],
\end{aligned}
$$

with $(n+1)$ denoting the time step $t+\Delta t \equiv(n+1) \Delta t$. While $D_{-}$denotes upwind differencing, $D_{0}$ stands for central differences, as are elucidated in figure 3 .

For numerical simulation of (18) in finite-volume formulation one can use arbitrary meshes, thus yielding

$$
\begin{aligned}
U_{i j k}^{(n+1)}=U_{i j k}^{(n)}-\frac{\Delta t}{\Omega_{i j k}}\left\{(\chi \cdot S)_{i+\frac{1}{2} \cdot j, k}+(\chi \cdot S)_{i-\frac{1}{2} \cdot j, k}+(\chi \cdot S)_{i, j+\frac{1}{2} \cdot k}\right. & \\
& \left.+(\chi \cdot S)_{i, j-\frac{1}{2} \cdot k}+(\chi \cdot S)_{i, j, k+\frac{1}{2}}+(\chi \cdot \mathbf{S})_{i, j, k-\frac{1}{2}}\right\}^{(n)}
\end{aligned}
$$

Using the flow variables and flux vectors in transformed coordinates $(\xi, \eta, \zeta)$ as defined in (19) the numerical simulation of the flow equations reduces to the following expression:

$$
\tilde{U}_{i j k}^{(n+1)}=\tilde{U}_{i j k}^{(n)}-\Delta t\left[\frac{D_{0}}{\Delta \xi} \tilde{F}_{i j k}+\frac{D_{0}}{\Delta \eta} \tilde{G}_{i j k}+\frac{D_{0}}{\Delta \zeta} \tilde{H}_{i j k}\right]^{(n)} .
$$

Cell-Centred Schemes

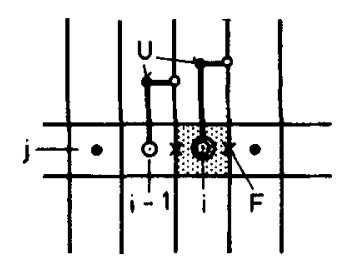

Upwind Schemes

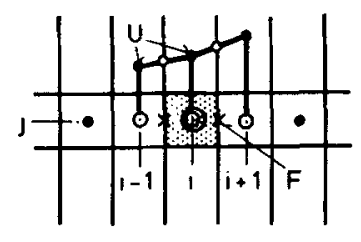

Central-Average Scheme
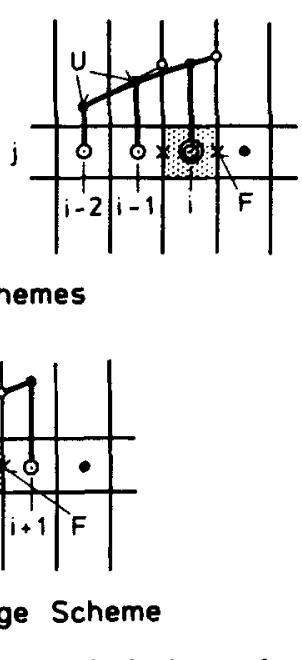

Figure 3. Standard numerical schemes for simulating the equations of flow fields.

\section{Cell-Vertex Schemes}
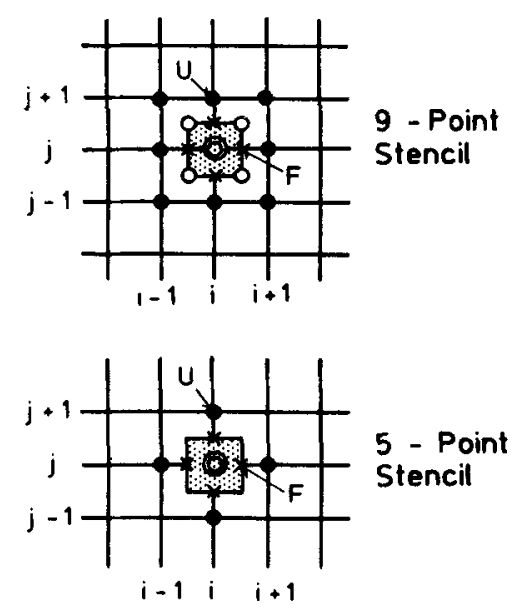
Thus (24) reproduces (21) exactly with $D$ replaced by $D_{0}$, confirming the equivalence of finite-difference and finite-volume methods.

The basic solution scheme of the viscous and nonviscous flow equations, which has been used in the present study, is a finite volume discretization with a Runge-Kutta integration in time, as is described by Jameson et al (1981). The original cell-centred code (Radespiel \& Kroll 1985) has been extended now to a cell-vertex code (Rossow 1989). Since the finite difference discretization for numerical simulation is based on the central difference scheme, which is insensitive to sensor sawtoothed spatial waviness of the field quantities, that may creep in due to odd-even point decoupling, an artificial dissipative term is added to the equation to damp out the high frequency oscillations. Hence, the numerical approximation to the integral equation (18) as expressed in (13) becomes extended to the form

where

$$
U_{i j k}^{(n+1)}=U_{i j k}^{(n)}-\frac{\Delta t}{\Omega_{i j k}}\left\{Q_{c}+Q_{v}+D\right\}_{i j k}^{(n)},
$$

$$
Q_{c}=\sum_{h} \delta_{h}\left(\chi_{c} \cdot \mathbf{S}\right), Q_{v}=\sum_{h} \delta_{h}\left(\chi_{v} \cdot \mathbf{S}\right), \text { with }(h \equiv i, j, k),
$$

denoting the balance of convective flux and of viscous flux, while $D$ is an artificial dissipative term. For the method of evaluation of the three flux terms of an elemental volume at a grid point $i, j, k$ of the flow field, one may refer to the details of the discretization procedure discussed by Radespiel \& Rossow (1990). In case of Euler equations for inviscid flows the flux term $Q_{v}$ in (25) drops out.

For the solution of (25) a five-stage Runge-Kutta time stepping scheme is used in the following way:

$$
\begin{aligned}
& U_{i j k}^{(0)} \equiv U_{i j k}^{(n)}, \\
& U_{i j k}^{(r)}=U_{i j k}^{(0)}-\alpha_{\mathrm{r}} \frac{\Delta t}{\Omega_{i j k}}\left[Q_{\mathrm{c}}+Q_{v}+D\right]_{i j k}^{(r-1)}, \\
& U_{i j k}^{(n+1)}=U_{i j k}^{(5)},
\end{aligned}
$$

where $r=1$ to 5 and the stage coefficients $\alpha_{r}$ are

$$
\alpha_{1}=1 / 4 ; \alpha_{2}=1 / 6 ; \alpha_{3}=3 / 8 ; \alpha_{4}=1 / 2 ; \alpha_{5}=1 \text {. }
$$

The artificial dissipation $D^{(r-1)}$ may be evaluated only for the 1st, 3rd and 5th stages of the scheme. The artificial dissipation model is based on the fluctuation of pressure as a sensor (Jameson 1981) defined by

$$
\bar{v}_{i j k}=\frac{p_{i-1, j, k}-2 p_{i j k}+p_{i+1, j, k}}{p_{i-1, j, k}+2 p_{i j k}+p_{i+1, j, k}} .
$$

For the evaluation of the dissipative operators in regions of normal grid structures and for highly stretched grids in the viscous layer one may refer to the details discussed by Radespiel (1989). The inclusion of the dissipative fluxes leads to the convergence of the procedure to a smooth solution. The stability of the numerical scheme was assured by choosing a CFL-no. of 9.5 both in the inner and outer block.

As steady state solutions are sought for, the use of several accelerating techniques 
is allowed to advance the solution. They are successive grid refinements, local time stepping, enthalpy damping, implicit residual averaging and finally the multigrid technique.

\section{Numerical study of complex flow fields around aerodynamical configurations}

The nonlinear partial differential equations describing the physics of a flow field are inherently complex and the physics itself becomes more complex when vortical flows, shock-waves or shock-viscous layer interactions come into being-especially in transonic and hypersonic speed regimes. Further cases of complex flows arise when the aerodynamical configurations themselves have involved geometries, as in the case of a complete aircraft configuration with wing, fuselage, nacelle and pylon and possibly with contrarotating propfans with highly swept blades. Numerical studies and analysis of such flow fields are becoming more and more common now. While the numerical solution of Euler equations is widely used to study the global features of complex flow fields, more effort is needed to analyse the details of the viscous effects, especially in regions of high flow gradients, which the solution of the Navier-Stokes equations depends on. The following examples will elucidate the findings from a number of interesting studies.

\subsection{Vortical flow fields around delta wings and a delta shaped wing-canard combination}

The flow field of a slender delta wing moving in a compressible medium offers an ideal example for the study of complex flows, extended over a speed range from subsonic through transonic to supersonic onflow velocities. This is due to the fact that at moderate to high incidence angles of the wing a large part of the flow field is involved with the formation of vortices spiralling over the upper surface of the wing, thus contributing substantially to an additional vortex-lift which is useful for an outstarding flight performance. The spiralling flow is established by calling in a loss of total pressure thus giving rise to vorticities in the flow. Besides which shock waves and shock viscous-layer interactions are common at high subsonic to supersonic onflow velocities. A further interesting feature is the breakdown of the spiralling vortices at high incidence angles, causing a loss in the vortex-lift. Thus a detailed study of the physics of such flows can lead to many interesting findings.

The delta wings selected for the numerical studies are depicted in figure 4 , their having undergone extensive experimental wind-tunnel tests (Brennenstuhl \& Hummel 1982; Drougge 1988; Bergmann et al 1990; Elsenaar \& Hoijemakers 1990; Oelker 1990). While for the delta wings $\mathrm{O}-\mathrm{O}$ grid topologies have been used, it proved to be simple to provide $\mathrm{H}-\mathrm{H}$ grid topology for the wing-canard combination and maintain the same grid structure for canard-on and -off configurations.

The numerical study with the simple delta wing has been undertaken by using the Euler equations, the solution of which yields all the essential field data which can be plotted and depicted as cross-flow fields or surface distributions of velocities and pressure, as have been shown in figure 5 . The trace of the spiralling vortices and the isolines of total pressure at a cross-cut reveal further details. In order to validate the computational data more closely with those of experiments the $c_{p}$-distributions 

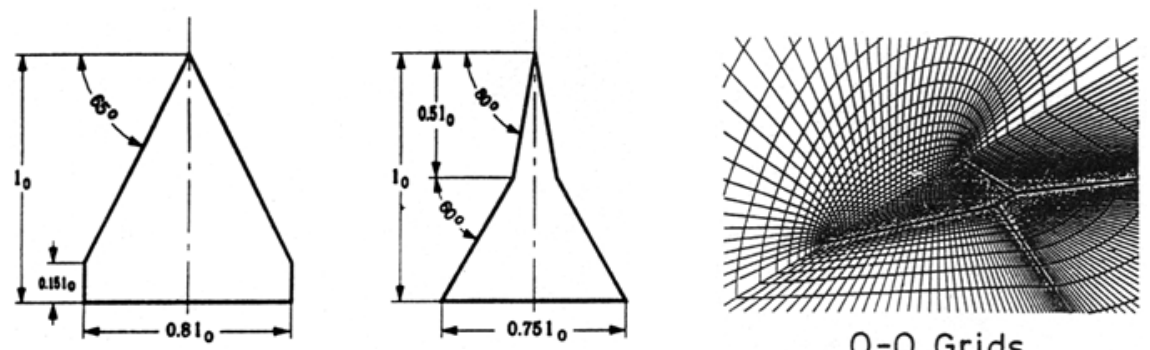

0-O Grids
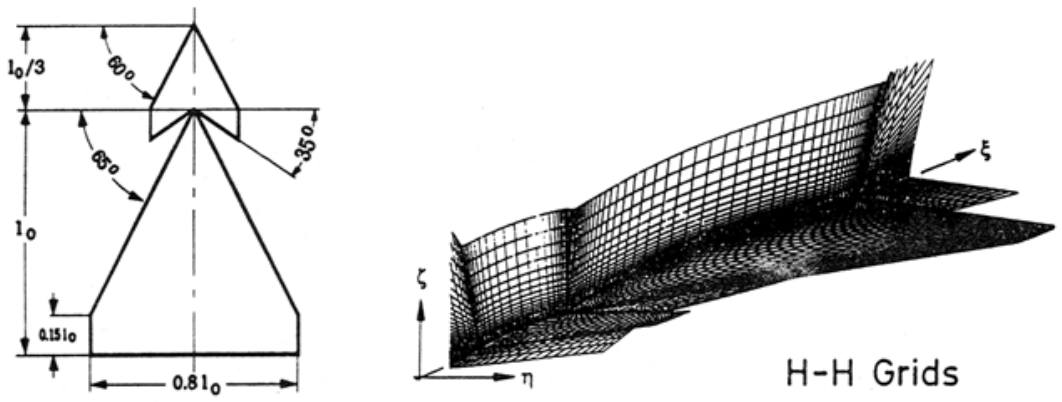

Figure 4. Delta wing configurations for numerical study of vortical flow fields by using Euler- and $\mathrm{N}-\mathrm{S}$ equations.
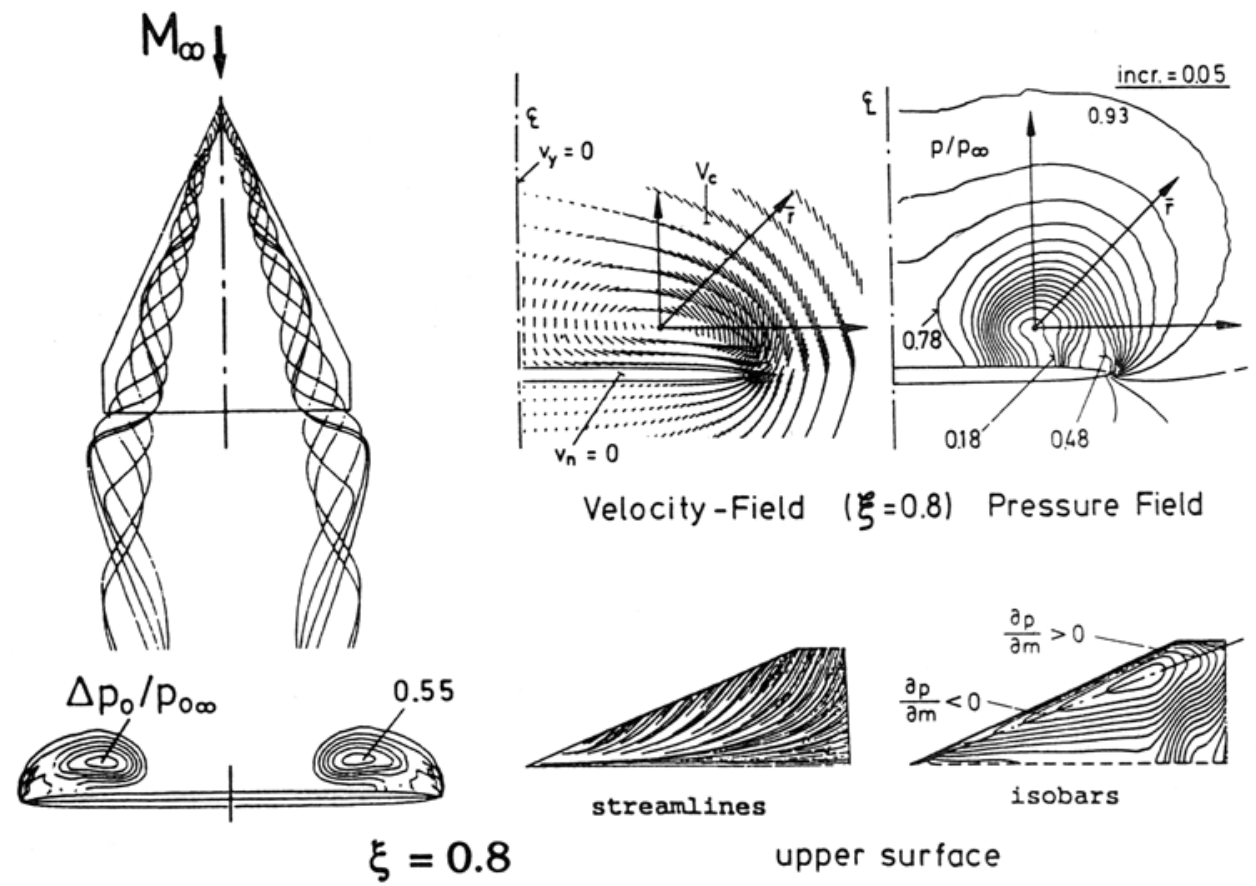

Velocity-Field $(\xi=0.8)$ Pressure Field
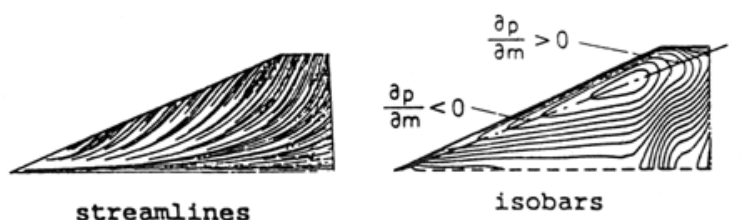

Figure 5. Numerical study depicting the cross-flow and surface-flow of a delta wing as yielded by the solution of Euler equations. $M_{\infty}=0.85 ; \alpha=20^{\circ}$. 

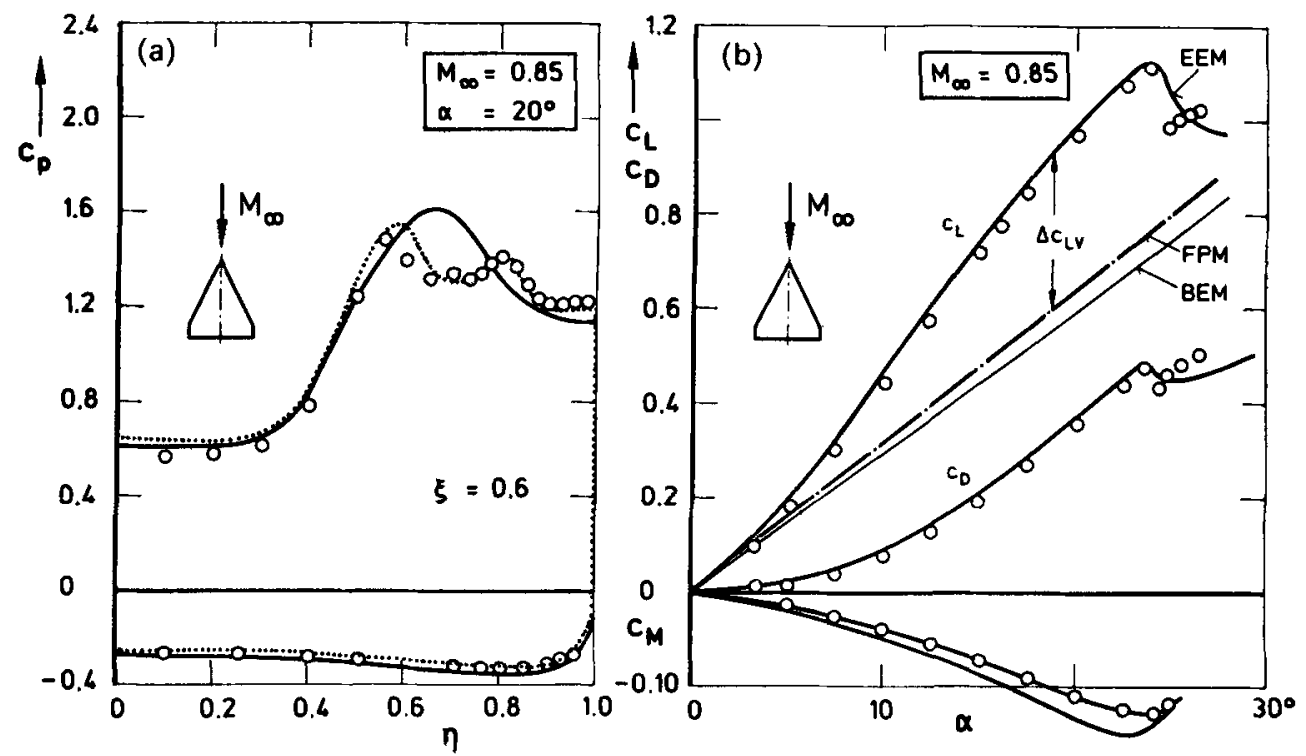

Figure 6. Comparison of numerical (Longo \& Das 1990) and experimental (Elsenaar \& Hoijemakers 1990) values of surface pressures (a) and aerodynamic forces (b) of the delta wing. In (a) $\bigcirc$ represent experimental values, - are values calculated using the Euler equation and .... are values from the Navier-Stokes equations. In (b) $O$ are experimental values while - are numerical values.

on the wing surface are compared in figure 6 and it was observed that the total lift and drag forces confirm very good agreement.

Having now the field data one can take up interesting analysis on the physics of the flow, regarding the setting in of the spiralling motion with loss of total pressure in it and on the changes in the structure of the vortical flow till its breakdown at
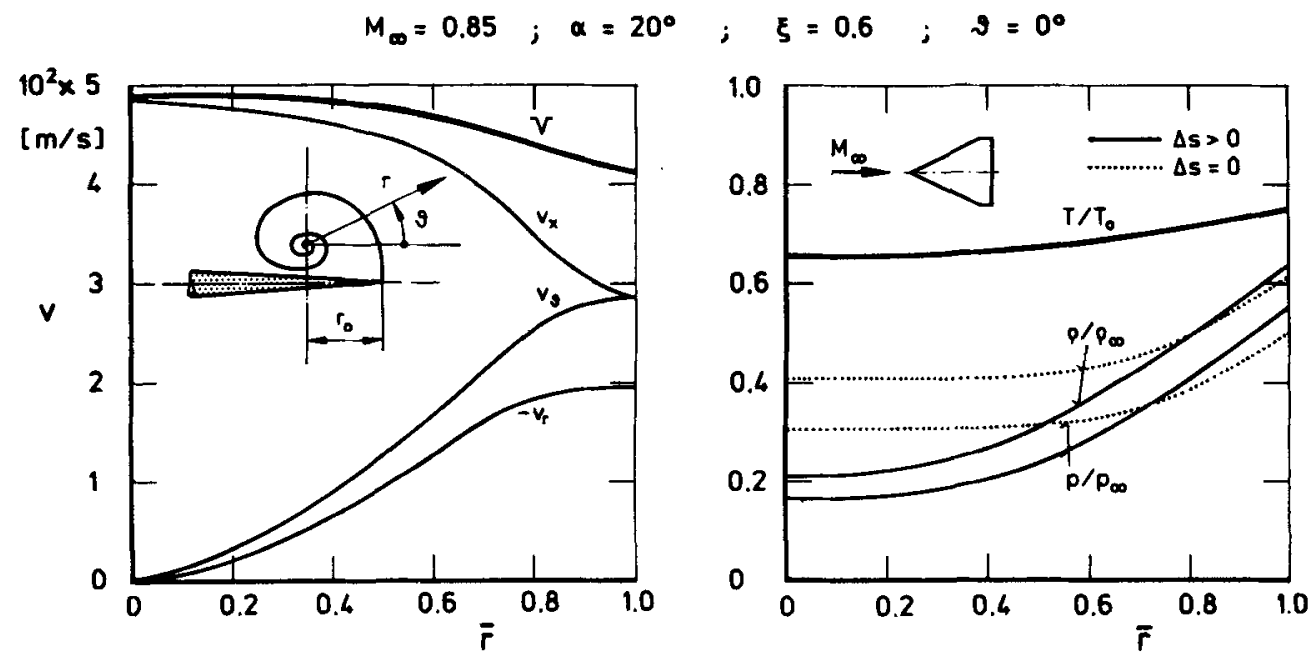

Figure 7. Pressure-, temperature- and velocity-distribution in the spiralling vortical-flow of a delta wing. 
(a)

$$
M_{\infty}=0.85 ; \quad \alpha=20^{\circ} ; \quad \xi=0.6 ; \quad \vartheta=0^{\circ}
$$
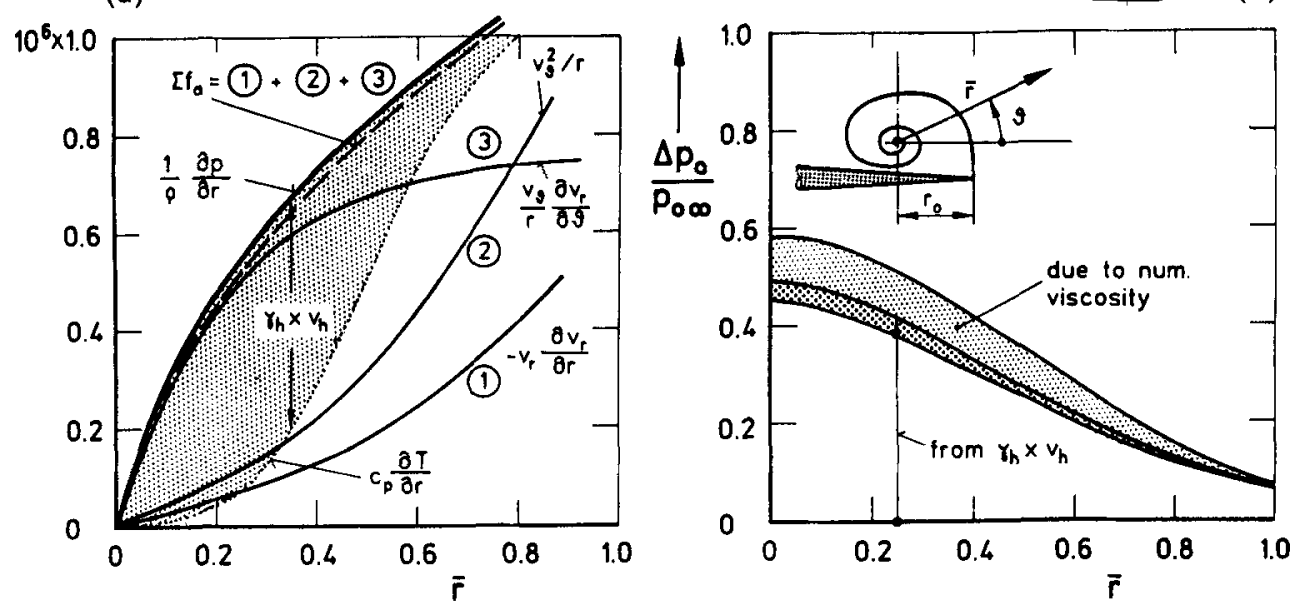

Figure 8. The pressure forces (a) and loss in total pressure (b) required for setting in the spiralling flow.

high incidence (Das 1991). With the imposed boundary conditions on the wing surface, and at the symmetry section of the wing the velocity and pressure distributions in the vortex take their typical runs, as are shown in figure 7 for a cross-flow plane $\xi=0.6$. It is evident that an isentropic pressure distribution corresponding to the velocity $V$ can by no means bring up the required accelerating forces to establish the spiralling - the flow has to be rotational, thus causing loss in total pressure for adjusting the radial forces which are needed. The nature of the accelerating forces and loss of total pressure along a radial line from the vortex core are depicted in figure 8 .

From the upper surface isobars of the delta wing as shown in figure 5 one can observe that an adverse pressure gradient appears in the rear part of the wing, which increases significantly at higher angles of incidence. The loss of total pressure and the adverse pressure gradient in the spiralling flow both increasing with the angle of incidence of the wing leads to the formation of two saddle points along the vortex axis - the one causes a reverse flow and the other contributes to high radial flow outward as has been depicted in figure 9 and is well elucidated (Das 1991). As a result the spiralling structure of the vortex breaks down, now causing a drop in the vortex-lift.

In order to improve the aerodynamic properties of delta wings various planforms have been investigated in the past, - one promising configuration being the strake - or double-delta wing. Numerical study on the strake wing shown in figure 4 was undertaken by using the Navier-Stokes equations and turbulent eddy viscosity modelling. A comparison of the pressure distributions and of the total forces and moments with the experimental values, as are shown in figure 10 , confirm the validation of both the results. Further comparisons of the surface flow and cross-flow of the wing as yielded by the numerical and experimental results in figure 11 prove the reliability of the method of calculation. For more examples of such studies one can refer to the cited literature (Das \& Longo 1994a). 
$\alpha=19.5^{\circ}$

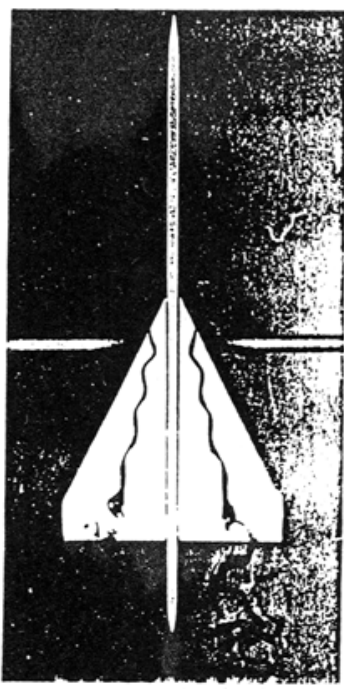

Num. Comp.: $M_{\infty}=0.4$
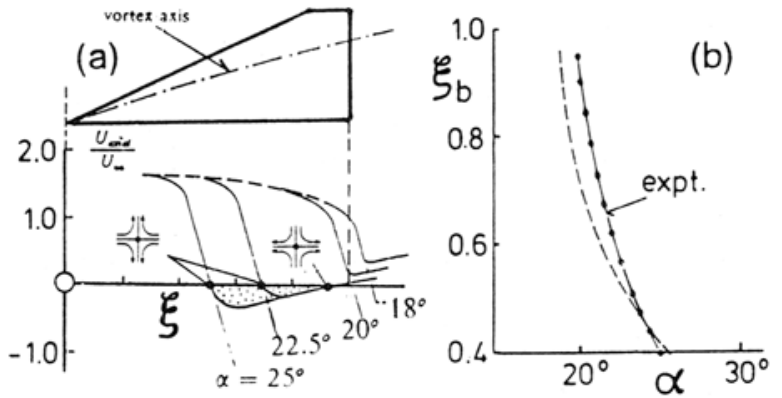

\section{Expt. $\left(M_{\infty}=0.1\right)$}

Figure 9. Analysis of the flow condition leading to vortex breakdown and loss of vortex-lift. (a) Flow reversal with saddle points and (b) vortex breakdown.
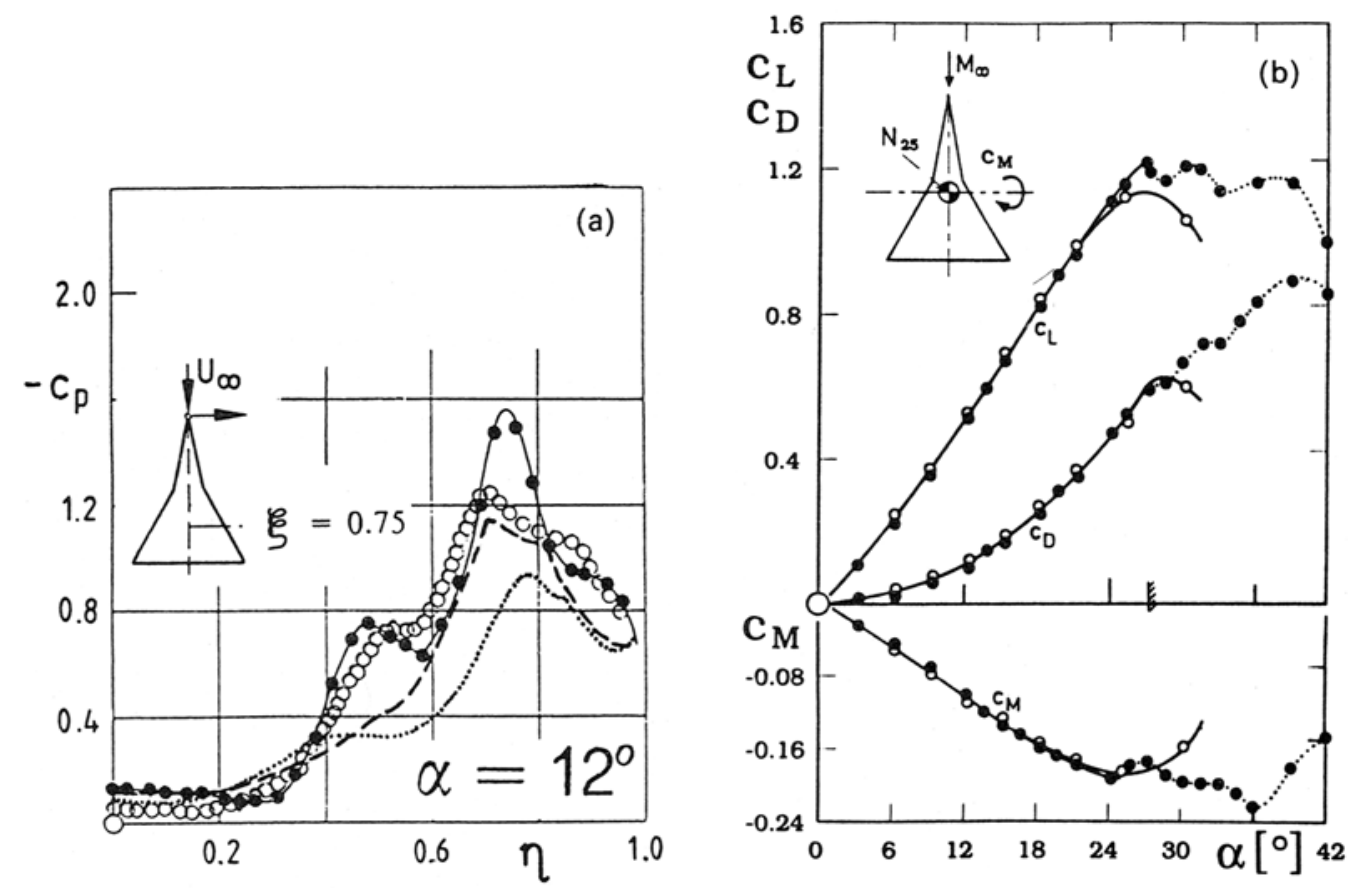

Figure 10. Surface pressure and aerodynamic force coefficients of the double-delta wing. (a) Cross-wise $c_{p}$ distribution [O experimental (Brennenstuhl \& Hummel 1982); $O$ present - numerical (Das \& Longo 1994a); - - - numerical (Krause \& Liu 1989); $\cdots$ numerical (Hartwich et al 1988) ]; (b) forces and moment $\left[\operatorname{Re}=1.33 \times 10^{6}\right.$; $\bigcirc$ numerical $\left(M_{\infty}=0.3\right)$; experimental $\left(M_{\infty}=0.1\right.$, Brennenstuhl \& Hummel 1982)]. 


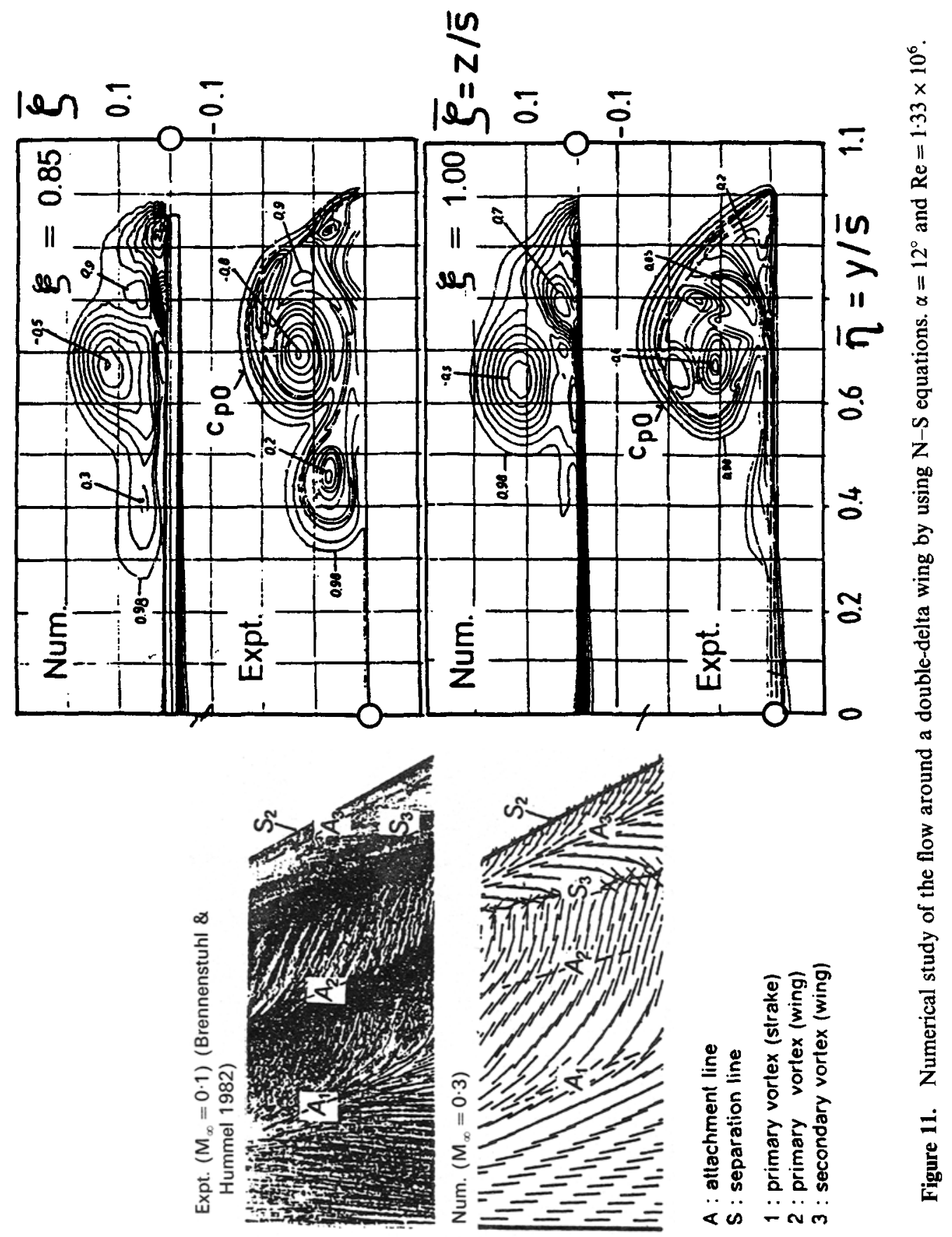



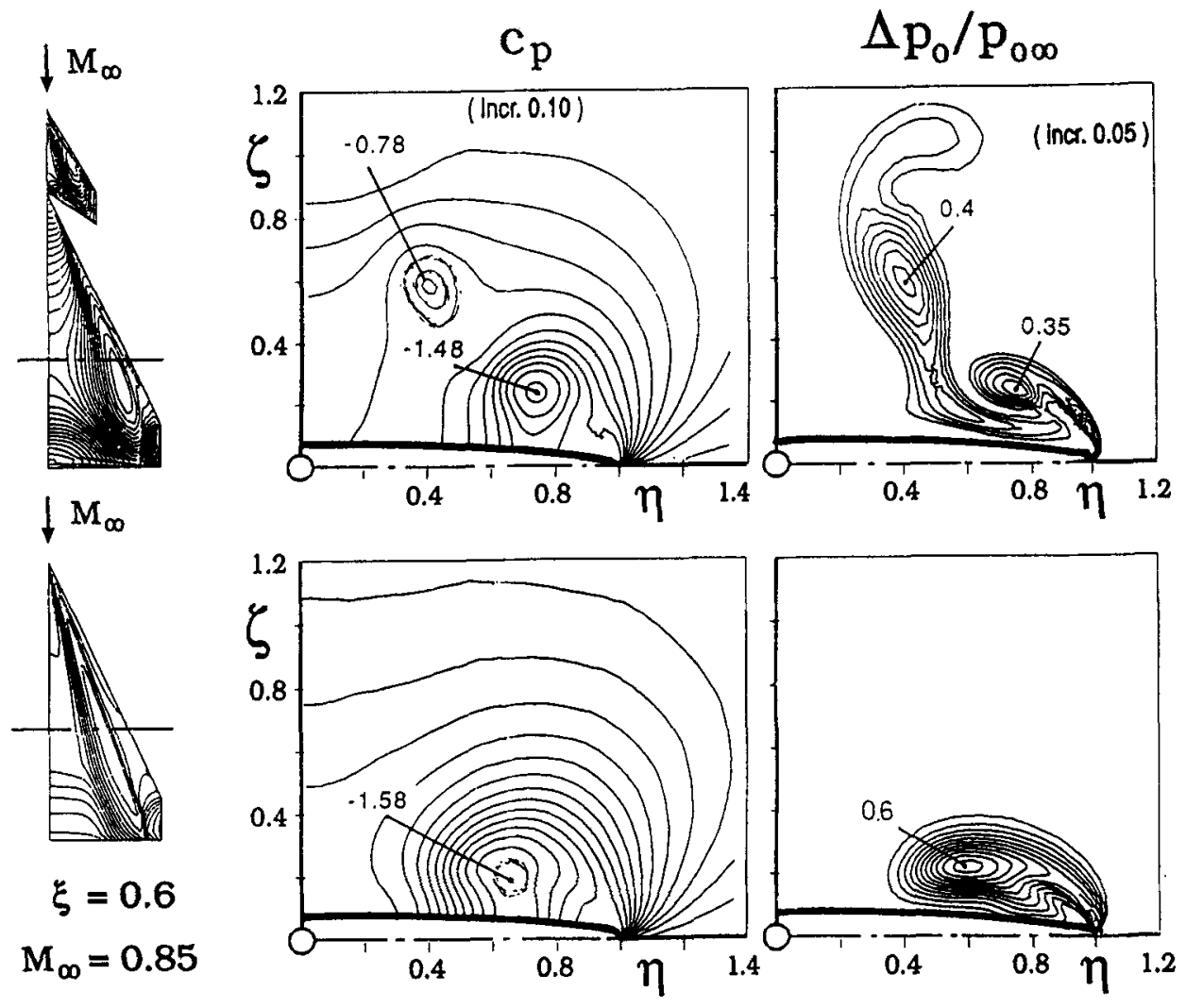

$\alpha=20^{\circ}$

Figure 12. The field quantities in the cross-flow plane of a wing-canard configuration as yielded by the solution of Euler equations.

It is known from wind tunnel measurements as well as flight tests that a delta shaped wing-canard configuration distinguishes itself through outstanding aerodynamic properties, primarily by maintaining its vortex-lift up to high incidence angles. Thus it offers an ideal example to analyse the complex flows due to multivortices spiralling over the wing.

So one can take up similar studies as with the simple delta wing already described above. The numerical field data have been plotted and depicted in a similar way, one example being shown in figure 12. The canard imparting downward momentum to the air particles ahead of the wing produces canard-lift and a decrease in the wing-lift due to the downwash created by it. Because of this and due to the canard vortex spiralling over the wing, the wing vortex becomes weaker having less loss in total pressure and also less adverse pressure gradient in the rear region. The loss of total pressure in the vortex core with the canard off and on has been compared in figure 13. Consequently, the spiralling vortex structure and the vortex-lift are maintained without breakdown up to high incidence angles, as is evident from the curves of total forces in figure 14. With a closely coupled canard the drop in the wing-lift is just compensated by the canard-lift; however, the lift curve $c_{L}(\alpha)$ continues its rise with the same slope up to higher incidence angles than the wing alone. 

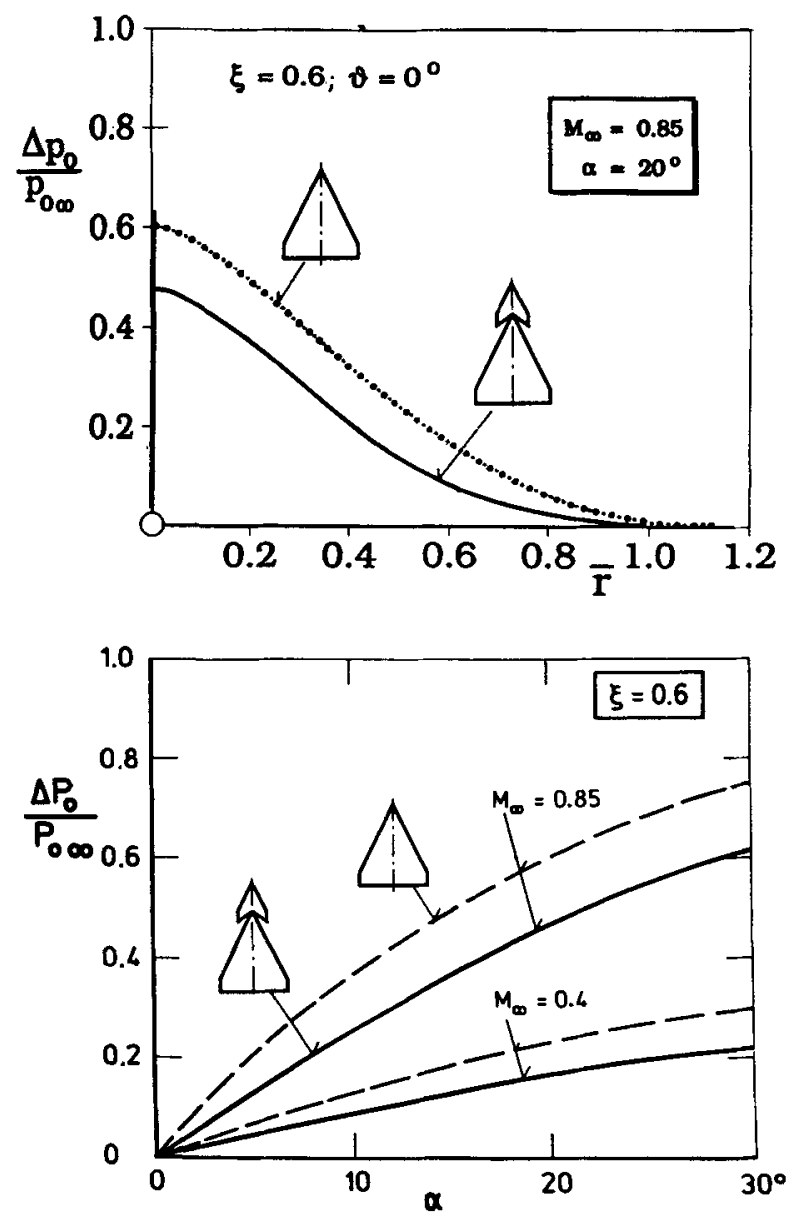

Figure 13. Loss of total pressure in the vortex core of a wing-canard configuration with the canard on and off.

Extensive analysis of the physics of the complex flow around this wing-canard combination has been presented in Das \& Longo (1994b).

\subsection{Flow fields around space vehicles}

Further studies on flow fields of slender delta wings at subsonic up to supersonic and hypersonic speeds concern the configurations used for space vehicles - the two present examples being the American space-shuttle and the European space-project HERMES, as are shown in figure 15 , both have extensive experimental data, as cited in the literature (Bornemann \& Surber 1978; Esch 1989; Radespiel \& Quast 1989). The recent measurements on a space shuttle model (Radespiel \& Quast 1989) comprise detailed investigations of surface flows and pressure distributions, as well as of total forces and moments. In order to reproduce all these the numerical studies were based on the Navier-Stokes equations with eddy-viscosity modelling of the turbulent viscous stresses (Baldwin \& Lomax 1978). A comparison of the numerical and experimental results is shown in figure 16 confirming excellent agreement. The details of the numerical method have been discussed by Das \& Longo (1994a). 


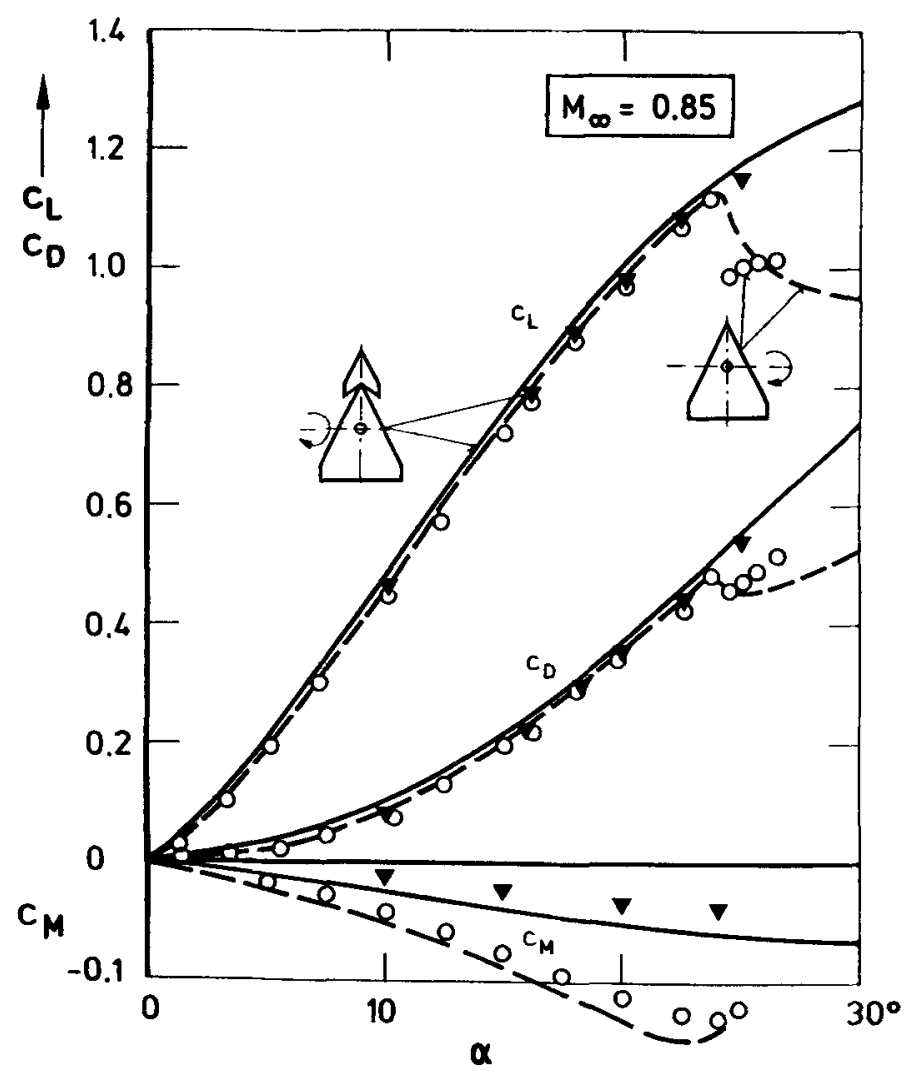

Figure 14. Aerodynamic ccefficients of total lift, drag and moment of a wingcanard configuration with the canard on and off. [- numerical (Das \& Longo 1994a); - - numerical (Longo \& Das 1990); $\nabla$ experimental (Elsenaar \& Hoijemakers 1990).]

Numerical studies on the HERMES-configuration were undertaken for flight conditions at supersonic Mach number $M_{\infty}=2.5$ to 8.0 for symmetrical and unsymmetrical onflows. Because of the complex flows over the wing and body, especially at unsymmetric onflows, it was essential to base the investigations on the solution of the Euler equations. The field data have been plotted as cross-flow velocities, cross-flow isobars and iso-Mach-lines as well as surface plots of isobars and streamlines. Finally the force coefficients $c_{L}, c_{D}$ and $c_{M}$ have been evaluated for symmetrical onflow as well as the stability derivatives at unsymmetrical flow conditions, as are depicted in figures 17 and 18. Comparison with the experimental results proves the outstanding reliability of the numerical methods used. For further details on this work one may refer to the paper by Schöne and coworkers (Schöne \& Bidault 1991; Schöne et al 1991).

3.3 Flow fields of large span wings including wing-fuselage and wing-nacelle interference

Most of the present transport airplanes with large span wings fly at transonic Mach numbers, such that the onflow velocity to the wings lies in the supercritical regime, 

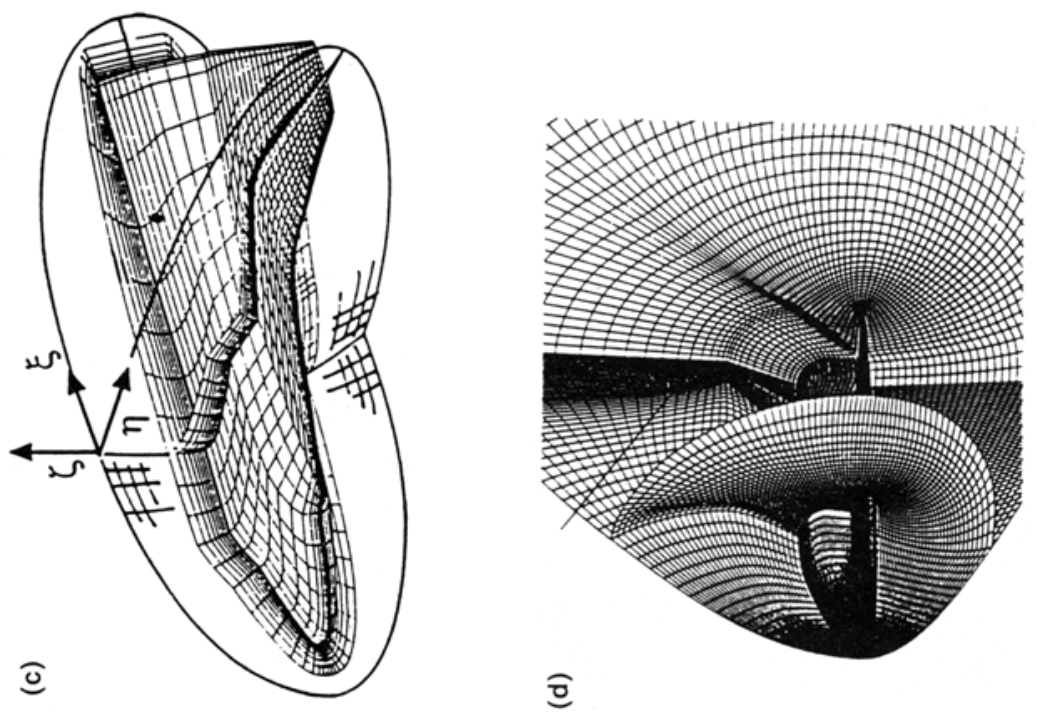

年
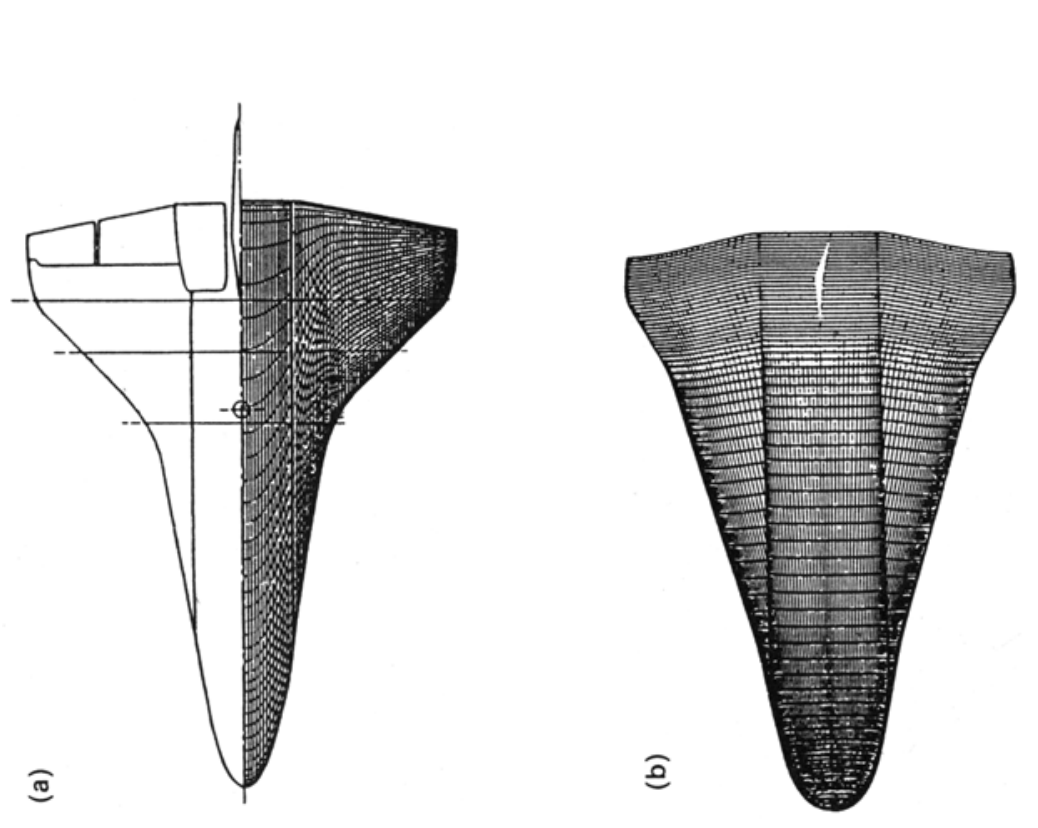

을

ลे

吾

焉

竧过

की

ตㄴ.

总总 

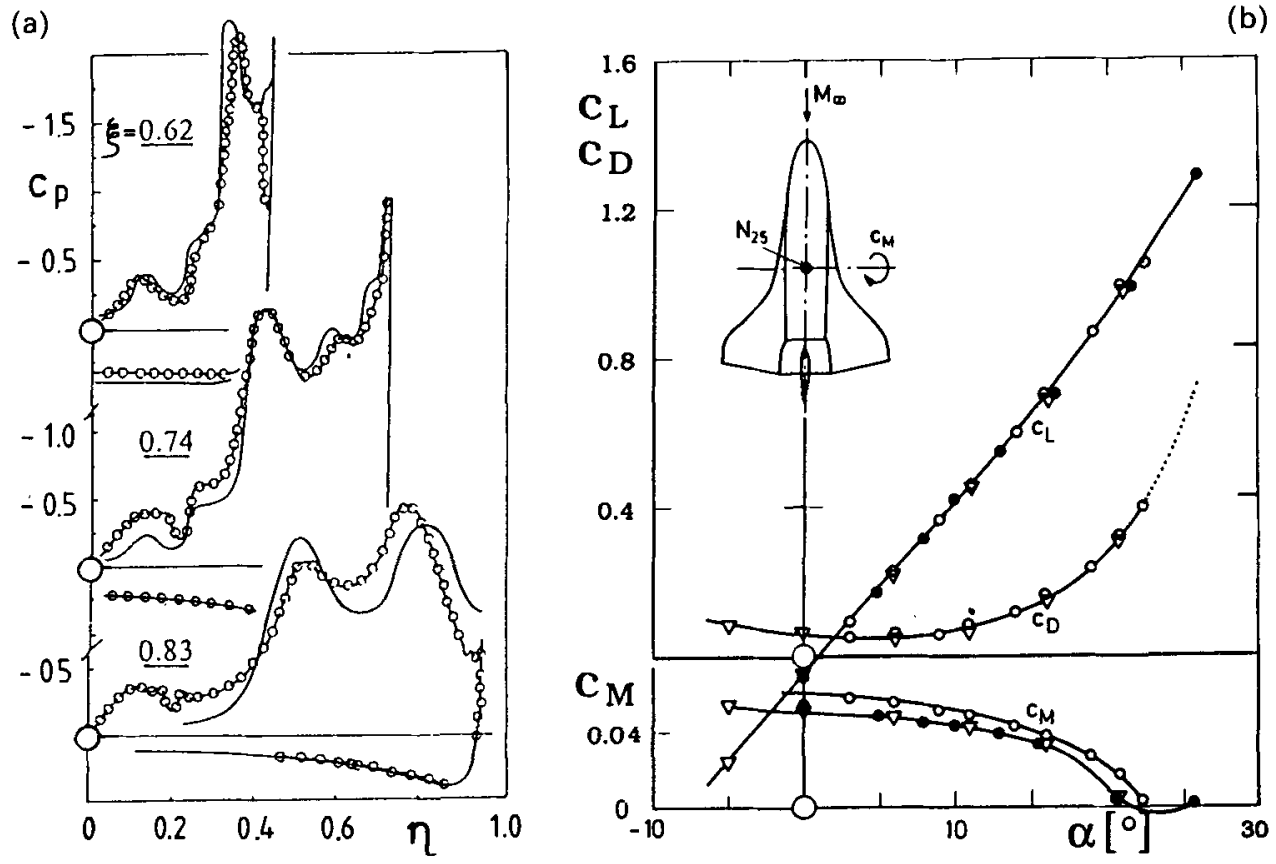

Figure 16. Pressure distributions and total forces and moments of the US-Space Orbiter at $M_{\infty}=0.4$ as yielded by the solution of $N-S$ equations. (a) Cross-wise $c_{p}$ distribution ( $\alpha=21^{\circ} ;-$ experiment; $-O$-numerical); (b) forces and moment $\left[\mathrm{M}_{\infty}=0.176 ; \mathrm{Re}=9 \times 10^{6} ; \bigcirc\right.$ numerical; 9 experimental (Bornemann \& Surber 1978); $\nabla$ experimental (Radespiel \& Quast 1989)].

thus creating local supersonic zones on the wing upper surface. As a result, shock waves and shock boundary layer interactions are very common at the cruising condition. The transonic flow being extremely sensitive to any changes in the boundary condition, also to the formation of boundary layer thickness, it is essential to consider the effect of viscosity on the surface flow of the wing. This can be realized by basing the numerical studies on the solution of Navier-Stokes equations or else combining the solution of Euler equations with iterative correction of boundary layer displacement thickness.

In the case of the wing alone it is essential to base the numerical studies on the solution of the Navier-Stokes equations with eddy-viscosity modelling of the viscous stresses, as has been undertaken by Radespiel (1989). Two examples are considered in the paper cited, one being the transonic flow around an aerofoil (RAE-2822) at $M_{\infty}=0.73, \alpha=2.79^{\circ}$ and $\operatorname{Re}=6.5 \times 10^{6}$, depicting the $c_{p}$-distribution on the wing surface as well as the skin friction on the suction side. The calculated values are in excellent agreement with the experimental results. The second example concerning the flow around an ONERA-M6 wing at $M_{\infty}=0.84, \operatorname{Re}_{\infty}=1.1 \times 10^{6}$ and $\alpha=3.06^{\circ}$ and $6.06^{\circ}$, will be taken up here to demonstrate the complex physics which may become involved. 


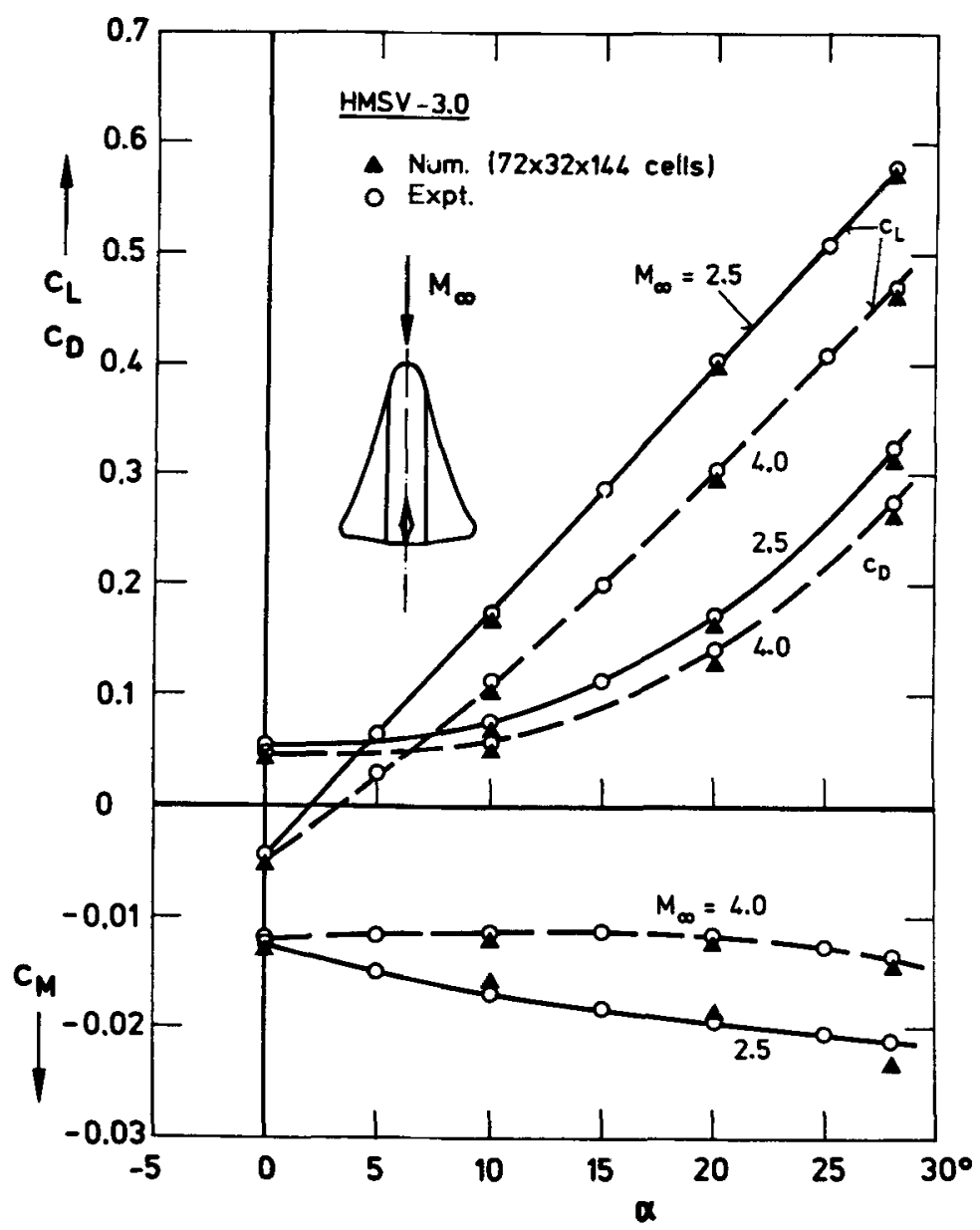

Figure 17. Numerical study of aerodynamic force and moment coefficients of the space orbiter Hermes by using Euler equations. [ $\Delta$ (Schöne \& Bidault 1991); O (Esch 1989).]

While for attached flow the numerical calculations performed with Baldwin-Lomax viscous modelling (Baldwin \& Lomax 1978) reproduce the $c_{p}$-distribution of the wind tunnel tests very closely, as shown in figure 19 for $\alpha=30^{\circ}$, one has to use the more complex Johnson-King (1984) modelling to have reliable results with separated flows as is evident from the results for $\alpha=60^{\circ}$. Both the numerical schemes prove themselves to be reliable and robust, the second method being however more complex and time consuming.

It will be now interesting to look into the interference effects of wing-fuselage configurations at high subsonic and transonic Mach numbers - at first by using the Euler equations. Extensive analysis has been undertaken for studying the flow fields of three basic configurations, which are shown in figure 20 . The flow field data are used to reproduce the surface flows and isobars as well as the lift distributions and total forces. Systematic variations of geometric and aerodynamic parameters have 

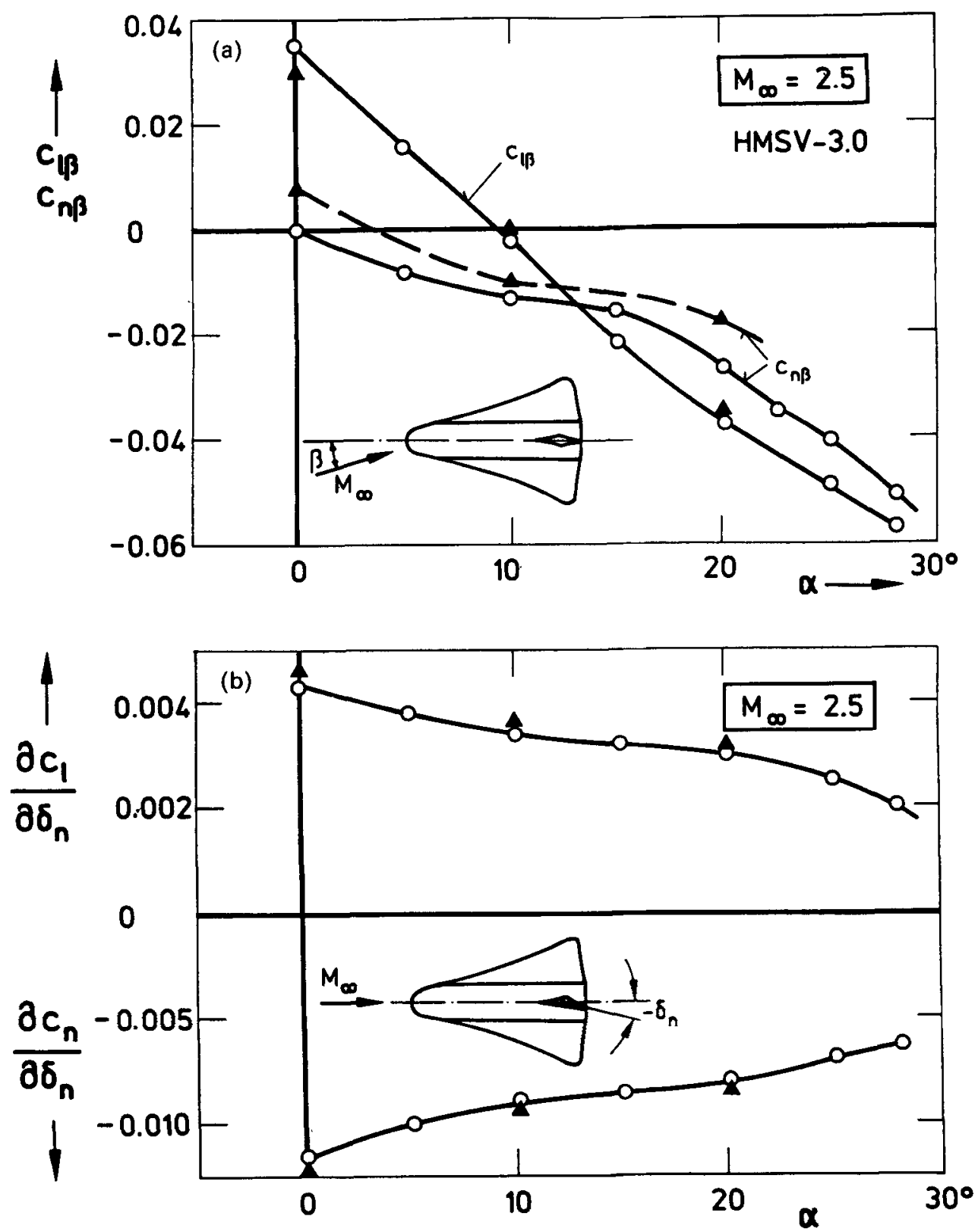

Figure 18. Analysis of the aerodynamic properties of the HERMES at the condition of side-slip (a) and rudder-deflection (b). [ $\triangle$ numerical (Schöne \& Bidault 1991); O experiment (Esch 1989).]

been undertaken for studying the nature of changes in the interference effects, especially for having higher aerodynamic efficiency of the configurations. Some essential results are depicted in figures 21 to 23. It is important to note that at transonic speeds one has to consider the viscous effects on the surface flows to get reliable results, for which some boundary-layer code has to be coupled to the numerical 

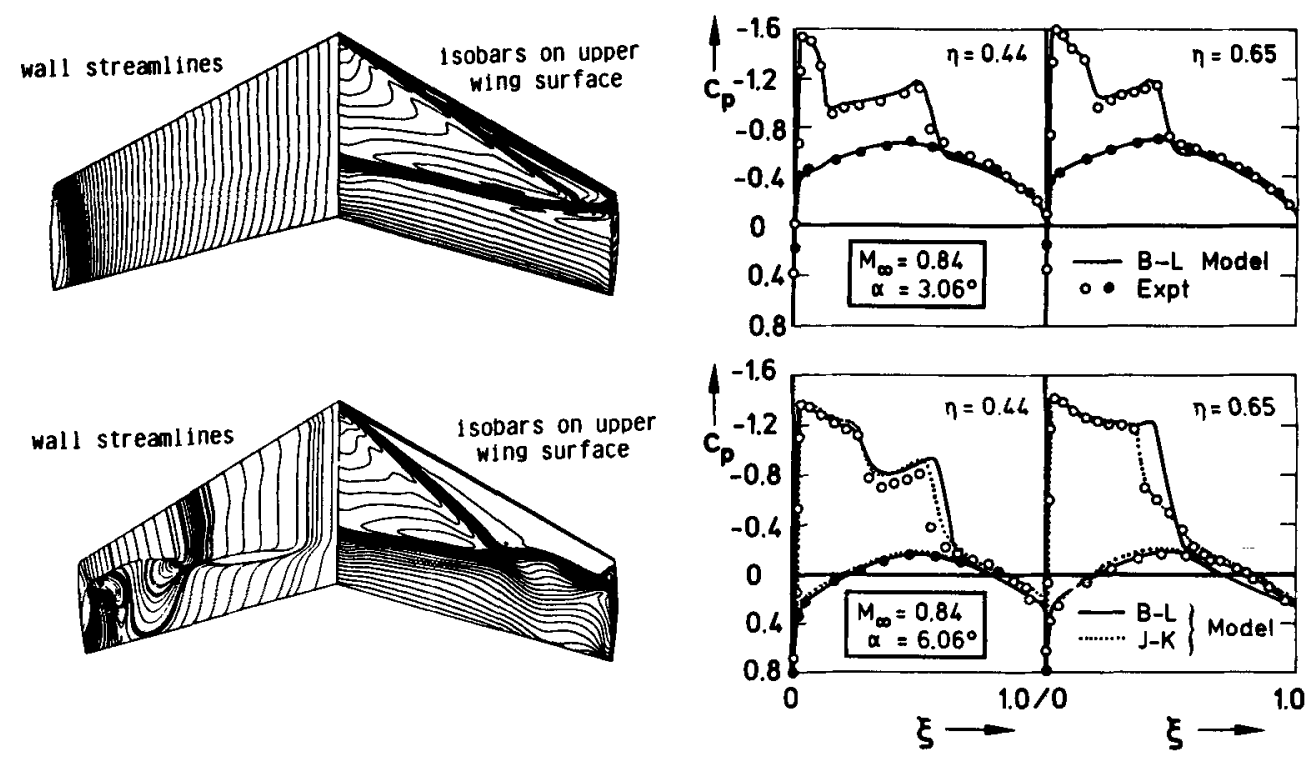

Figure 19. Numerical study of the flow-field of a transonic wing by using N-S equations. [B-L - Baldwin \& Lomax (1978); expt. - Schmitt \& Charpin (1979); J-K - Johnson \& King (1984).]

scheme treating inviscid flows. For all details of the numerical methods which have been followed in the above examples one can refer to the thesis by Wichmann (1992). It is evident that numerical studies using Navier-Stokes equations can give more reliable results quite directly - they are however more time consuming and hence expensive for comprehensive studies. A few test cases have been performed (Longo 1992) yielding very good results.

The numerical studies are finally aimed at determining optimum aerodynamic configurations of complete aircraft, including wings, fuselage, engines, nacelles and pylons, especially for achieving high values of lift to drag ratios for maximizing the parameter $\mathrm{M}_{\infty} c_{L} / c_{D}$ at transonic speed regimes. Although the effect of viscosity brings in significant changes in the surface flow of a super-critical aerofoil and thus to the upper surface pressure distributions, it was decided to make the numerical calculations by using the Euler equations as a preliminary study of the global features of the flow around the complex configurations. The viscous corrections for the sensitive wing surface flow can be done by coupling a numerical code for boundary layer flows.

The aircraft configuration with engine, nacelle and pylon is shown in figure 24 along with the sectional marking for close study of the $c_{p}$-distributions. The computational studies also yield extensive results as surface streamlines and isobars as well as field values of pressures and velocities as isoline plots. From the surface $c_{p}$-values some plots of spanwise lift distributions are demonstrated showing the aerodynamical interference effects resulting from the engine, nacelle and pylon. For further results one may refer to Rossow \& Ronzheimer (1991), Rudnik (1991) and Rossow et al (1992). 

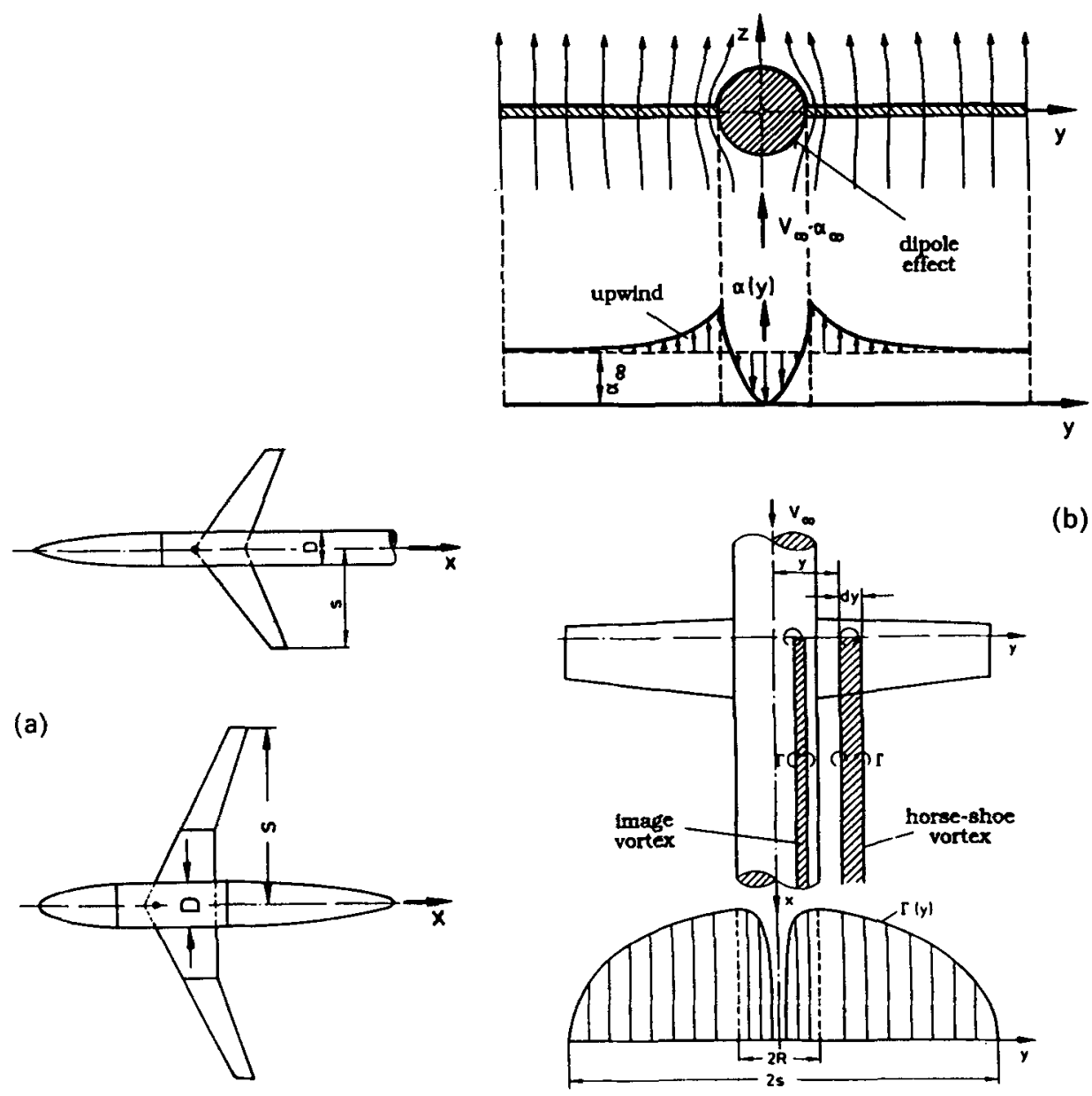

(b)

Figure 20. Wing-body combinations for numerical analysis of aerodynamic interference-effects by using Euler equations. (a) Wing-body configurations; (b) interference effects (idealized models).

\section{Numerical study of flow fields around propellers and helicopter rotors}

The modern aircraft-propellers and helicopter-rotors having transonic onflow conditions at the outer regions of the blades need extensive analysis for improved layout of the blade sections as well as of the blade shapes. In order to capture all the details of the flow, especially the appearance of shock waves, shock-boundary layer interactions and the vortical flow round the tips, it is essential to undertake numerical studies by using the Navier-Stokes equations. However, as a preliminary step the computational analysis was based on the Euler equations and two cases have been taken up, for which enough experimental data were available for validating the numerical results. These are a two-bladed propeller having tip advance ratio of $\Lambda=0.73$ with tip helical Mach number $\mathbf{M}_{h}=0.56$ and a hovering helicopter rotor with tip Mach number of $\mathrm{M}_{h}=0.79$. 

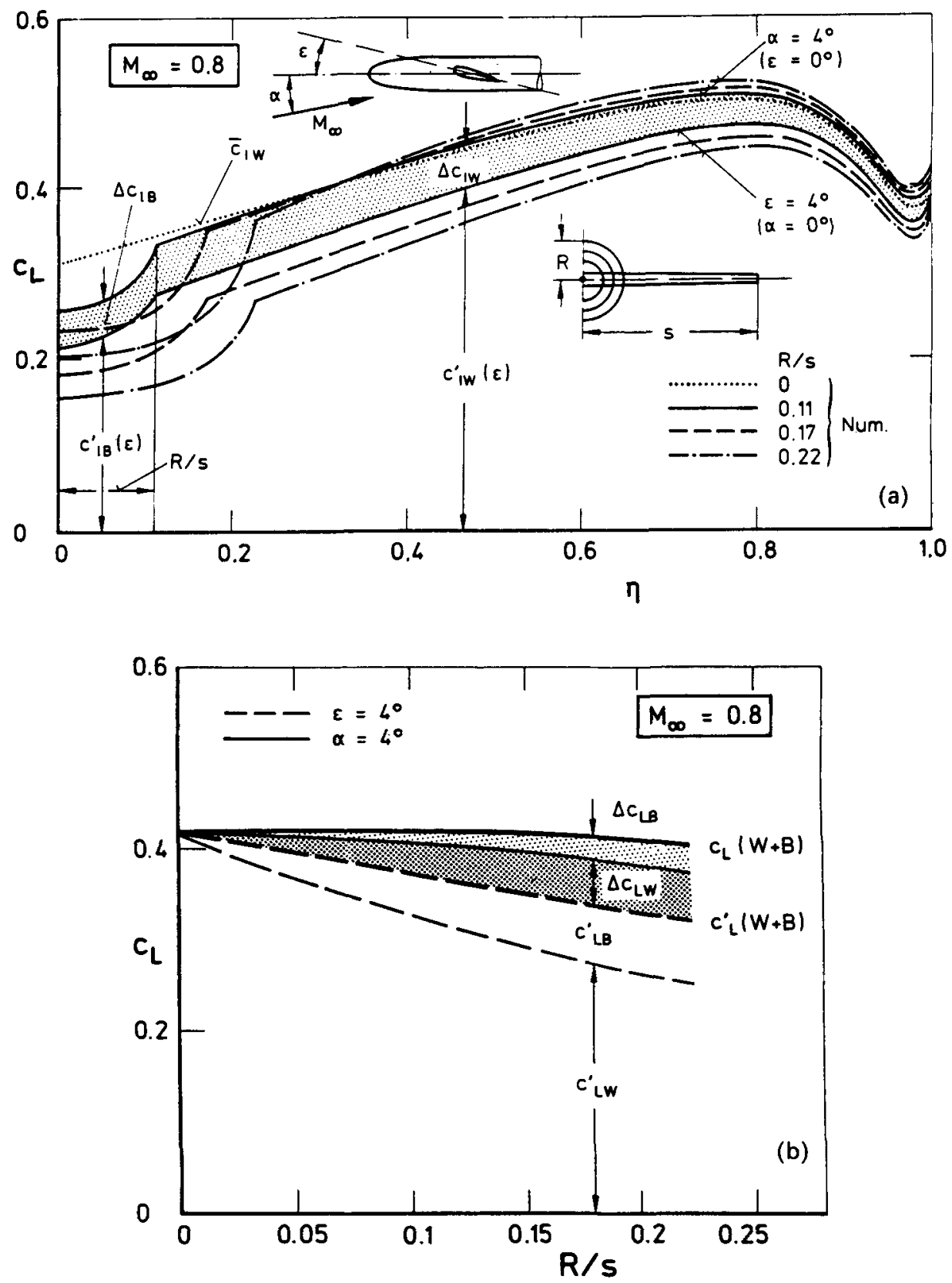

Figure 21. Analysis of the aerodynamic properties of wing-body combinations as function of wing-setting angle [numerical values of $R / s$ from Wichmann (1992)] (a) and body thickness (b).

From the numerical computations using $0-0$ field grids round the blades one obtains all the necessary field data for undertaking plots of blade loadings and blade surface flows, as well as of the tip vortices. The $c_{p}$-distributions on the blades are shown in figures 25 and 26 confirming very good agreement between the computed and 

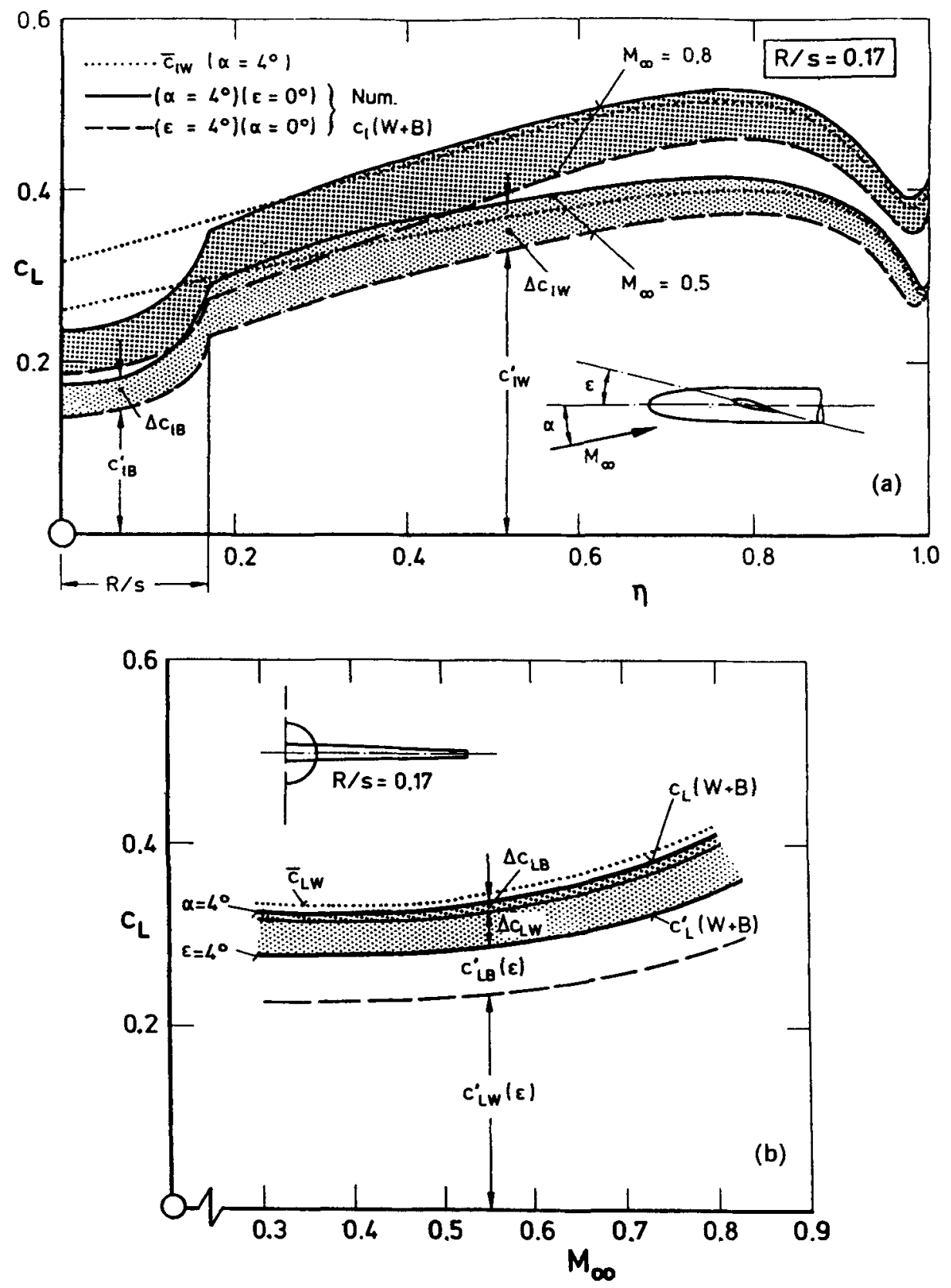

Figure 22. Effect of Mach-number on the spanwise lift-distribution (numerical values from Wichmann 1992) (a) and total-lift (b) of a wing-body combination. 

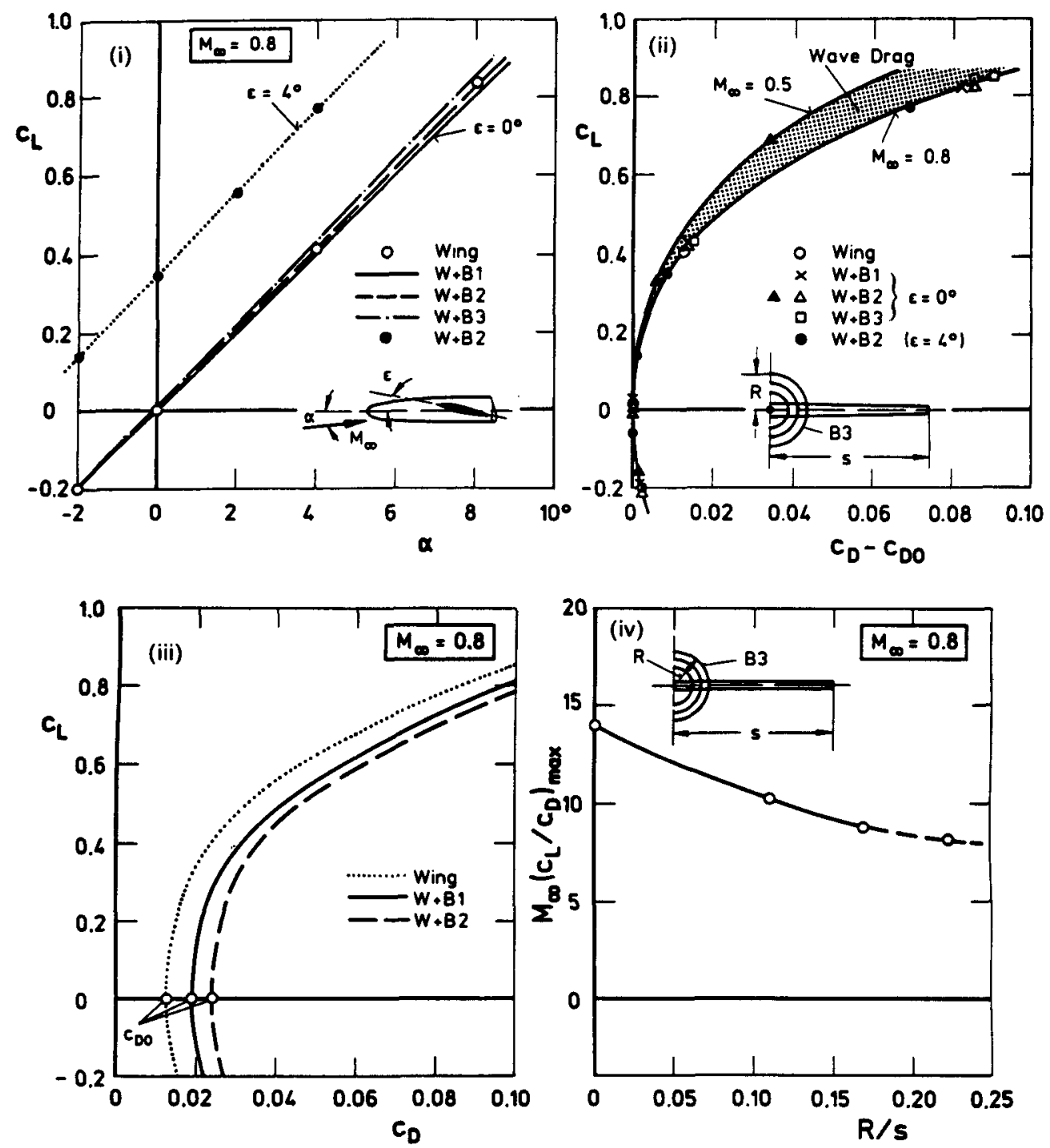

Figure 23. Numerical analysis of the maximum lift to drag ratio of wing-body combinations at the transonic Mach number of $\mathbf{M}_{\infty}=0 \cdot 8$. (Numerical values in (iii) from Wichmann 1992.)

measured data. For the details of the computational method using rotating reference frame for the Euler equations one can refer to the original thesis work (Kroll 1989). The above analysis will be useful both for aerodynamics and aeroacoustics.

\section{Numerical study of some unsteady flow fields}

Some common examples of unsteady aerodynamics are oscillating and plunging motions of aerofoils and wings, both having arbitrary steady forward motion. In recent years extensive studies have been undertaken on the unsteady motions of 

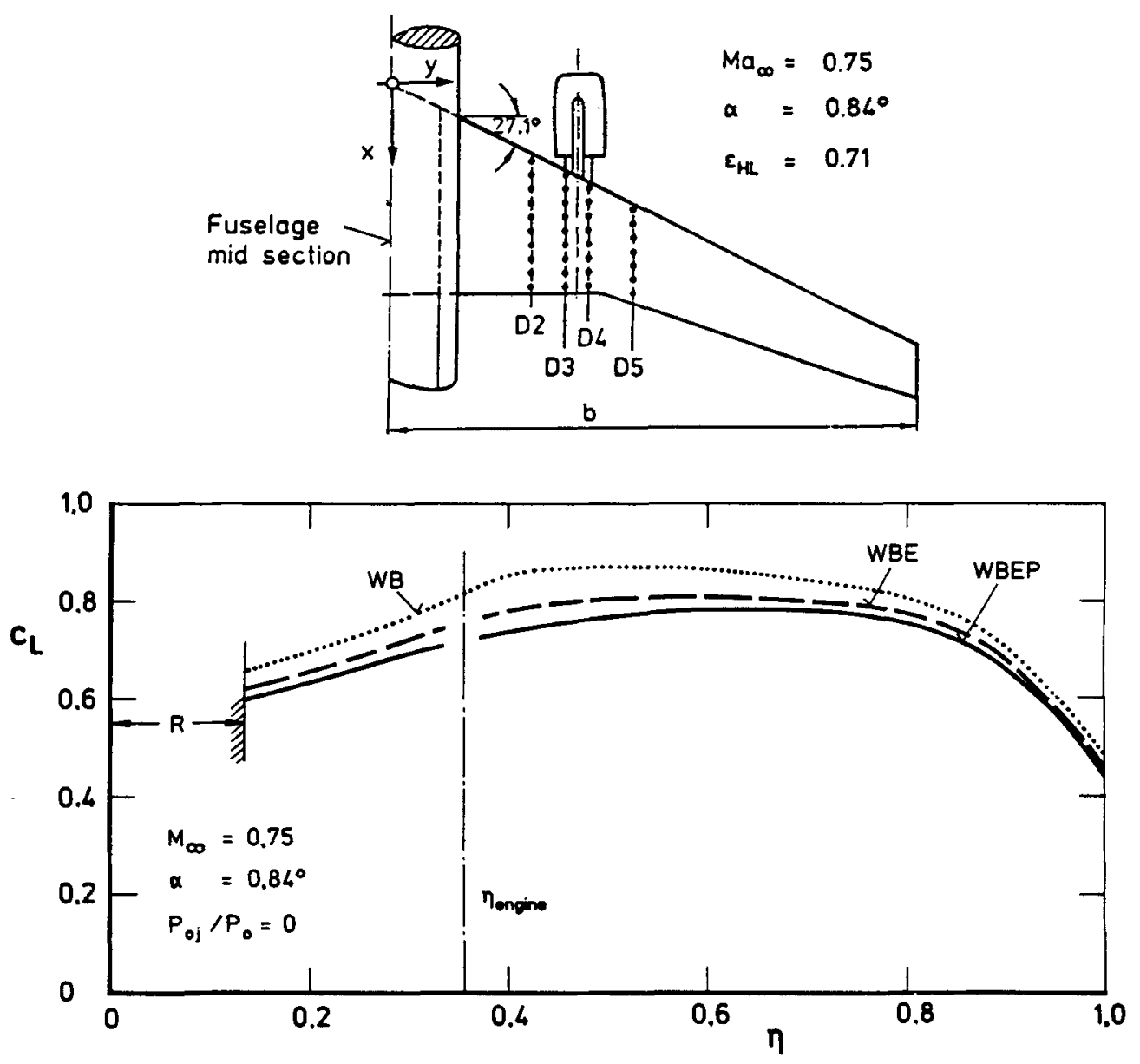

Figure 24. Numerical study of the changes in the spanwise lift distribution of a wing-body due to engine-installation.

aerofoils and wings by using numerical solutions of the Euler equations and validating the results with experimental data. Some numerical results on the unsteady forces and moment of an oscillating aerofoil are shown in figure 27 demonstrating the typical hysteresis effects.

Both with regard to aerodynamics and aeroacoustics much effort is now focused on the detailed study of unsteady flow fields of lifting helicopter rotors in forward motion. However, it seems essential to divide the numerical and experimental studies into three distinct stages:

(a) Flow field of a two-bladed nonlifting rotor in forward motion.

(b) Flow field of a two-bladed lifting rotor in forward motion.

(c) Flow field of a lifting multi-bladed rotor in forward motion.

The first case being much simpler than the other two will help to develop the numerical technique and some basic concepts before taking up the full problem with complex motion of the blades. 


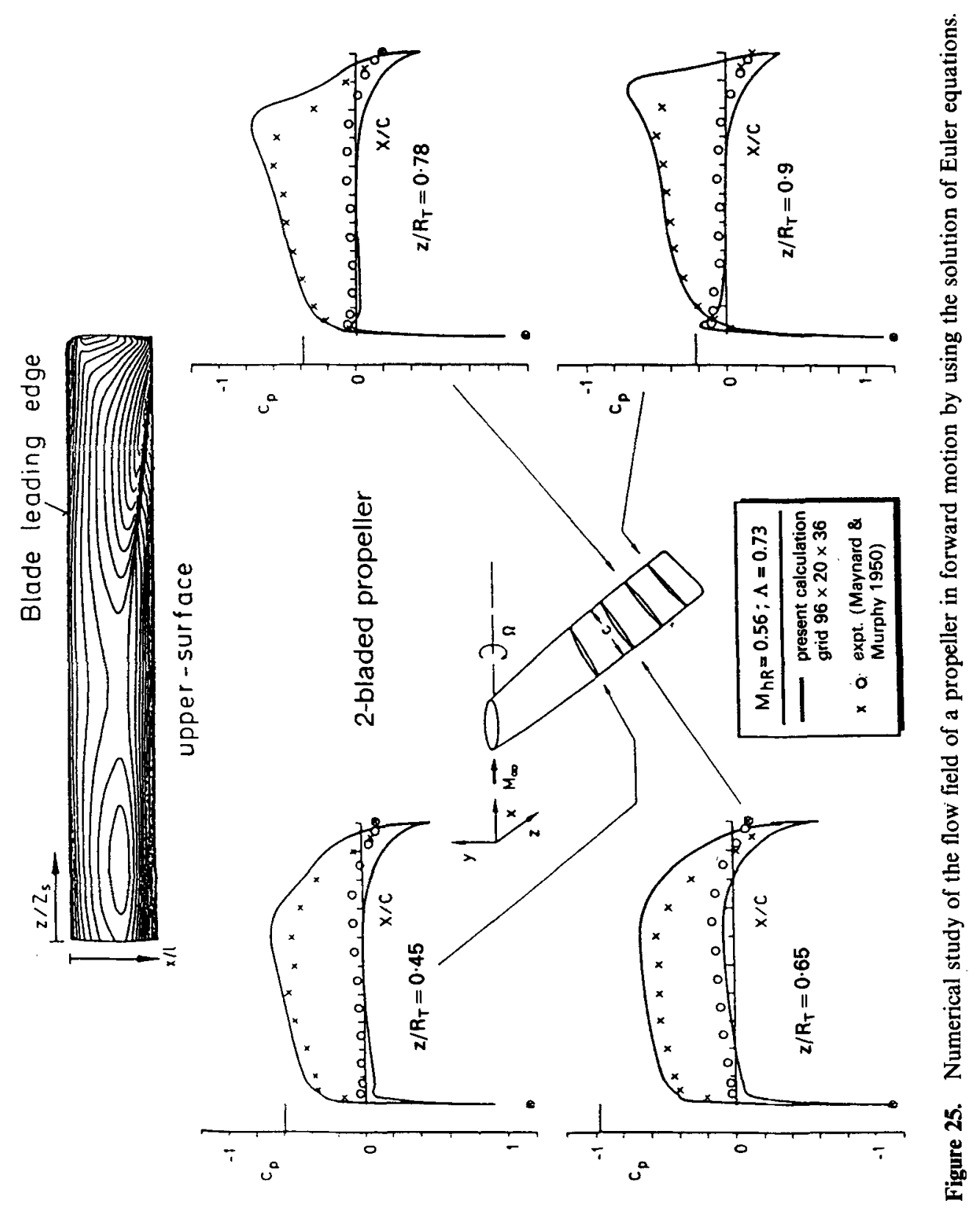




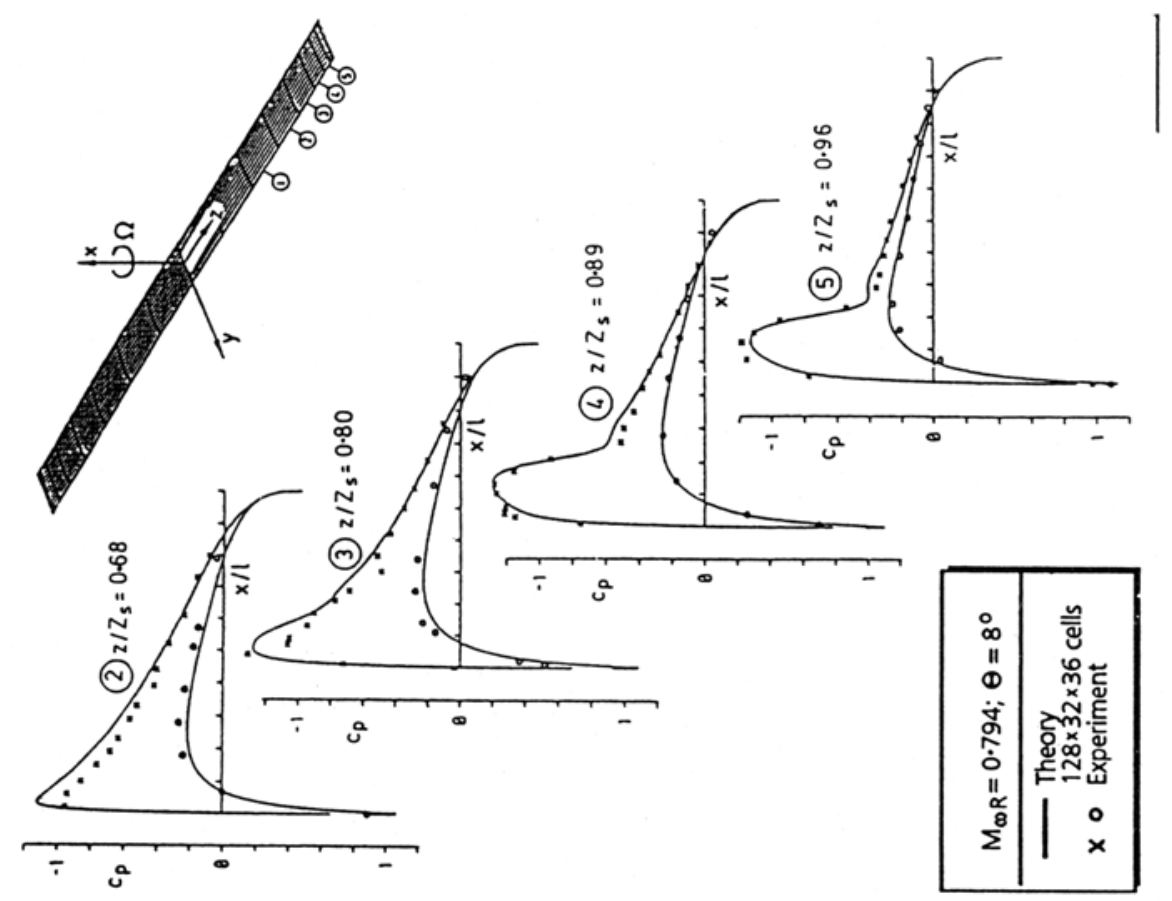

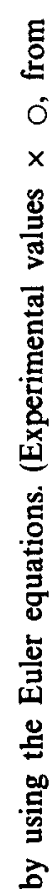
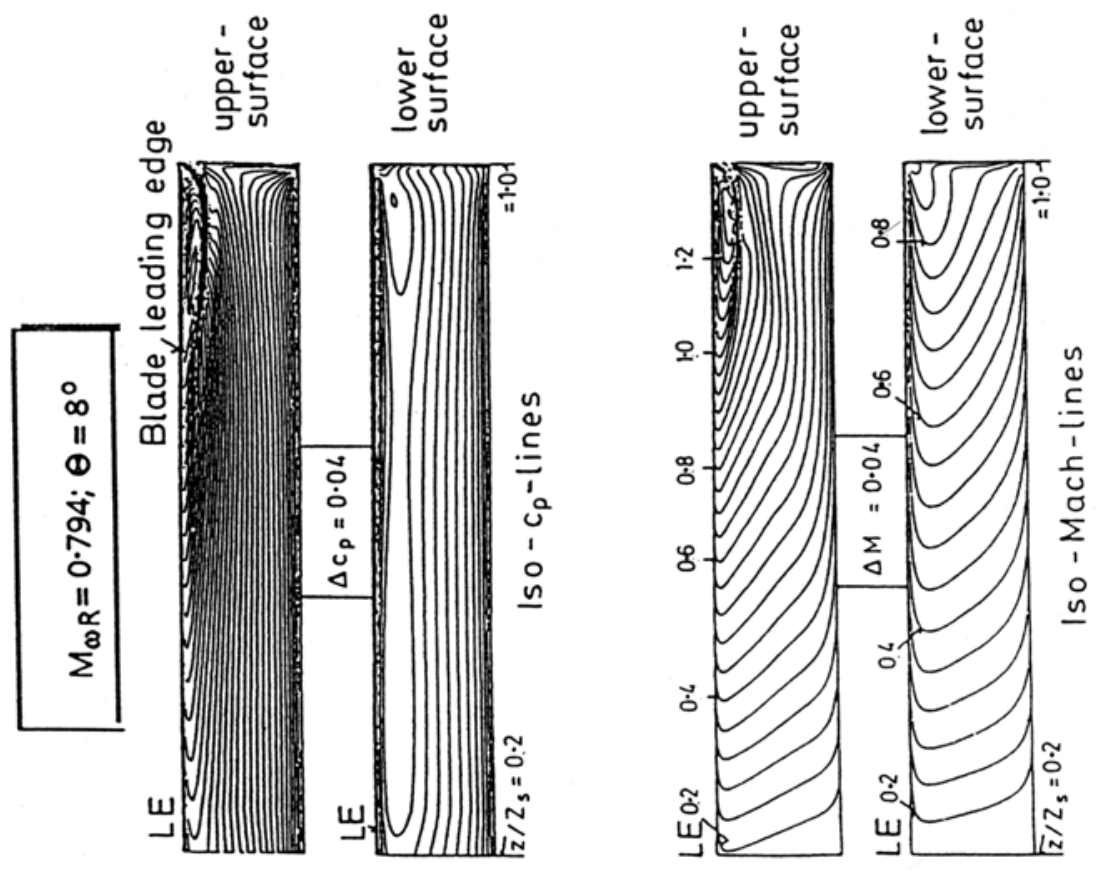

홍

.

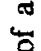

흔

음

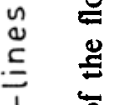

ป

i $\quad$ 离

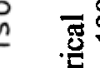

总骂

Z

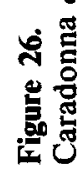




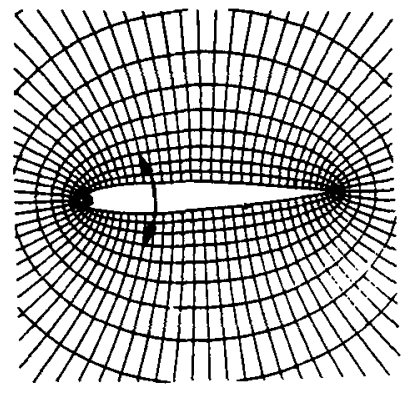

(a)

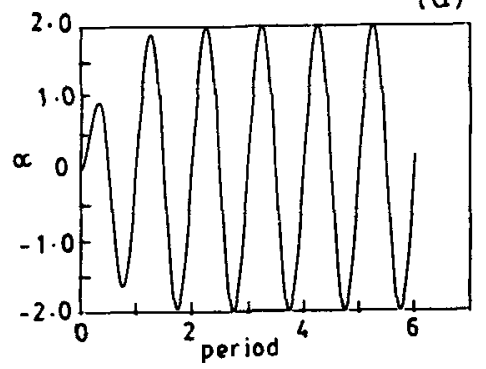

(c)

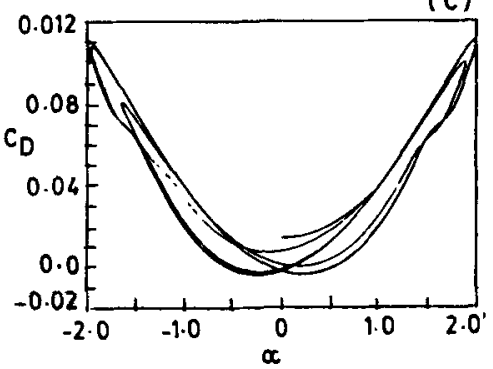

$M_{\infty}=0.755$

$\omega=0.1454$

$\mathrm{k}=0.0814$

$\alpha=2^{\circ}\left[1-e^{-\sigma t}\right] \sin \omega t$

\section{Aerofoil: NACA 0012}

(b)

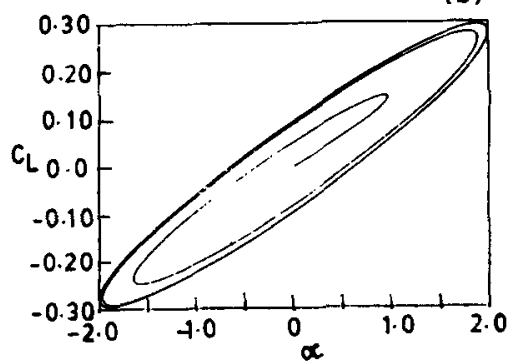

(d)

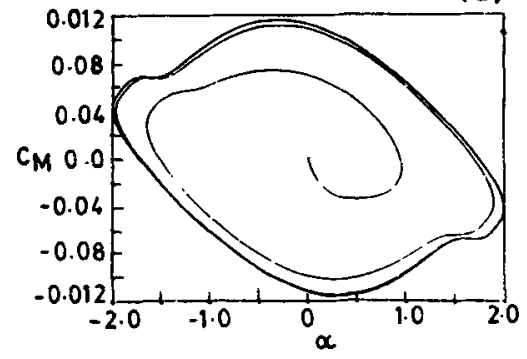

Figure 27. Numerical study of the unsteady flow field of an oscillating aerofoil by using the Euler equations.

Having the experimental data of $c_{p}$-distributions at the outer part of a nonlifting rotor blade, some comparisons of the numerical and experimental values at three sections with $r / R=0.855$ to 0.946 have been undertaken of which a few plots are shown in figure 28 confirming acceptable agreements. This work is being continued now as a doctorate thesis for studying the lifting cases (b) and (c).

\section{Conclusions}

The numerical methods for the solution of nonlinear partial differential equations being now well established, it has given a big impetus for undertaking detailed analysis of complex flow fields, which may arise from involved physics of the flow or due to the complexity of the geometrical configurations. Although it is preferable to base 

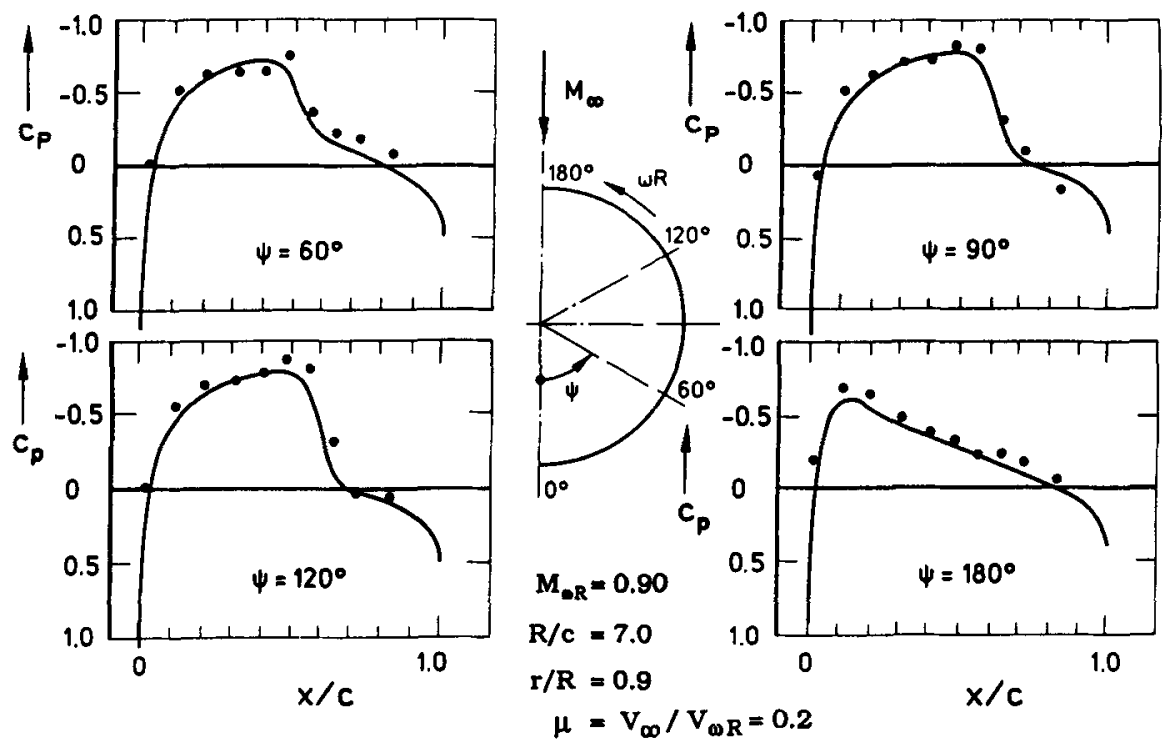

Figure 28. Numerical study of the unsteady flow field of a nonlifting helicopter rotor in forward motion by using the Euler equations. [3-D Euler code $\mathrm{O}-\mathrm{H}$ grids $(65 \times 17 \times 31)$; Expt: Caradonna et al (1984)].

the numerical studies on the solution of Navier-Stokes equations, they may not be highly useful if the efficiency and accuracy of the algorithms need still further effort for further improvement. Especially in case of extensive studies with systematic variations of aerodynamical and geometrical parameters it may be quite suitable to work with the numerical solutions of the Euler equations to obtain all the global features of the flow fields. The viscous effects are primarily confined in the regions of surface flows, especially where high cross-gradients come into being. While for complex flows around slender delta wings and bodies, as well as for space vehicles the numerical analysis based on the Euler equations yields quite acceptable results, one has to incorporate the effect of viscosity in the solution of the Euler equations when large span wings at supercritical onflows are concerned-and this can be usually done by coupling a boundary layer code to correct for the boundary layer displacement thickness.

Detailed analysis of the unsteady flow fields, which arise around counter-rotating propfans and helicopter rotors in forward flight have been taken up now by using the solution of Euler equations.

When further progress has been achieved, with the multigrid technique, applicable also for multiblock body-fitted grids around complex configurations, direct analysis with the Navier-Stokes equations will be more in use.

\section{List of symbols}

$a, a^{*} \quad$ local and critical speed of sound;

A Jacobian matrix;

$b \quad$ span of a wing; 


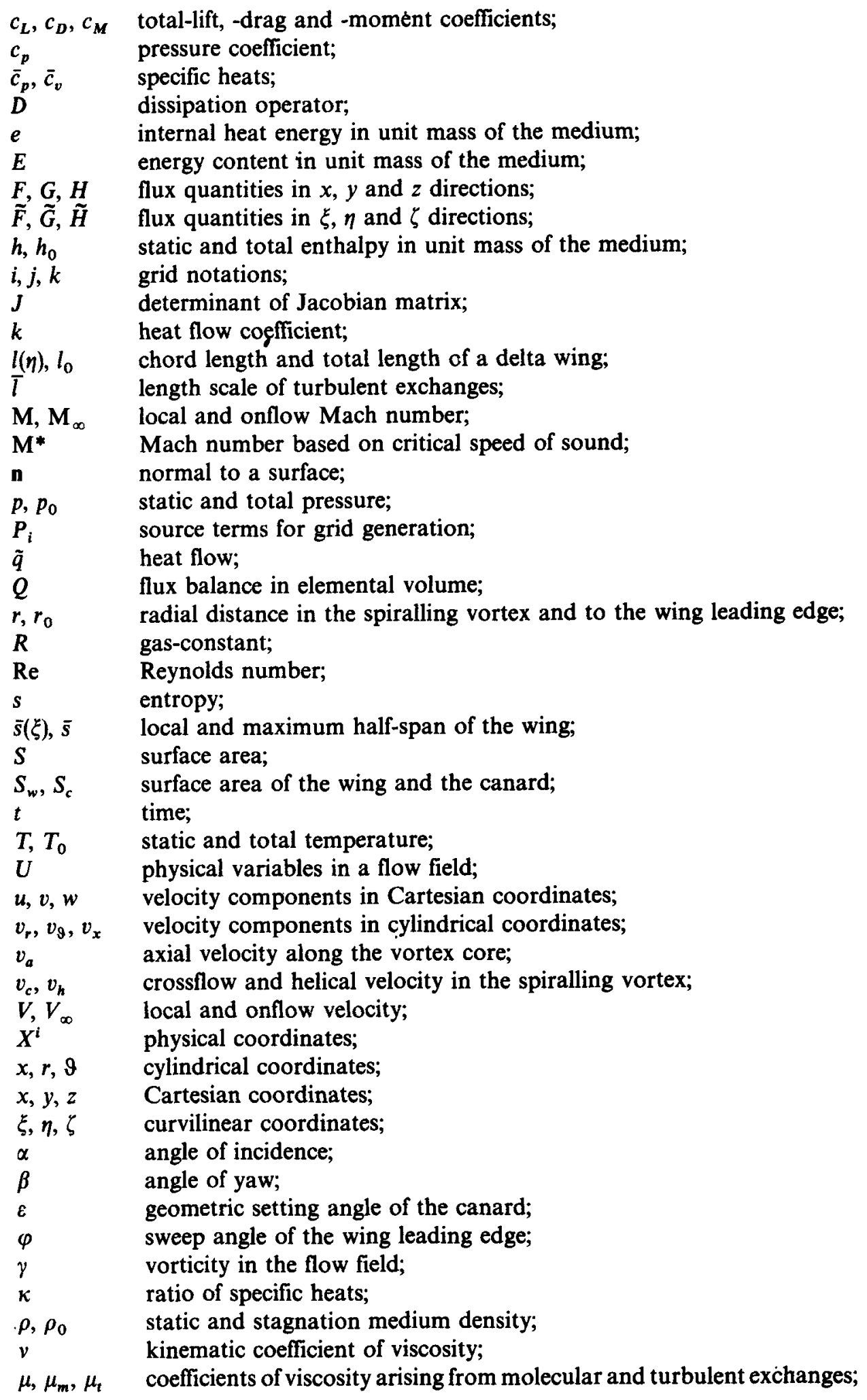


$\sigma_{v} \quad$ stress tensor due to viscosity;

$\mathbf{\Omega}, \tilde{\Omega} \quad$ volume of a cell element in Cartesian and curvilinear coordinate system; flux tensor in the flow field.

\section{References}

Agrawal S, Barnett R M, Robinson B A 1990 Investigation of vortex breakdown on a delta wing using Euler and Navier-Stokes equations. AGARD fluid dynamics panel symposium on vortex flow aerodynamics. AGARD-CP-494, pp. 24.1-24.12

Baldwin B S, Lomax H 1978 Thin layer approximation and algebraic model for separated turbulent flows. AIAA paper 78-257

Beam R M, Warming R F 1976 Upwind second order difference schemes and application to aerodynamics. AIAA J. 14: 1241-1249

Beam R M, Warming R F 1978 An implicit factored scheme for the compressible Navier-Stokes equations. AIAA J. 16: 385-402

Bergmann A, Hummel D, Oelker H -Chr 1990 Vortex formation over a closed coupled canard-wing-body configuration in unsymmetrical flow. AGARD Fluid Dynamics Panel Symposium on Vortex Flow Aerodynamics, AGARD-CP-494, pp. 14.1-14.14

Blazek J 1994 Verfahren zur Beschleunigung der Lösung der Euler- und Navier/Stokes Gleichungen bei stationären Über- und Hyperschallströmungen. Dissertation der Technische Universitat, Braunschweig

Blazek J, Kroll N, Radespiel R, Rossow C -C 1991 Upwind implicit residual smoothing method for multi-stage schemes. AIAA paper 91-1533

Bober L J, Chaussee D S, Kutler P 1983 Prediction of high speed propeller flow using a threedimensional Euler analysis. AIAA paper $83-0188$

Bornemann W E, Surber T E 1978 Aerodynamic design of the space shuttle orbiter. AGARD-CP-247, pp. 11.1-11.24

Brennenstuhl U, Hummel D 1982 Vortex formation over double-delta wings. ICAS Proc. vol. 2, pp. 1133-1146

Caradonna F X, Laub G H, Tung C 1984 An experimental investigation of the parallel bladevortex interaction. NASA-TM-86005

Caradonna F X, Tung C 1981 Experiments and analytical studies of a model helicopter rotor in hover. NACA TM-81232

Carstens V 1990 Die Berechnung von instationären kompressiblen Strömungen um schwingende Profile mit Hilfe eines Euler-Upwind Verfahrens. DLR-Festschrift, Göttingen, 1990

Chaussee D S 1986 High speed viscous flow calculations about complex configurations. AGARD-CP-412, pp. 29.1-29.17

Chaussee D S, Rizk Y M, Buning P G 1984 Viscous computation of a space-shuttle flow field. Lecture Notes in Physics-9th Int. Conf. on Numerical Method in Fluid Dynamics 218: $148-153$

Courant R, Friedrichs K O, Lewy H 1928 Uber die partiellen Differential-gleichungen der Physik. Math. Ann. 100: 32-74

Courant R, Isaacson E, Rees M 1952 On the solution of nonlinear hyperbolic differential equations by finite differences. Commun. Pure Appl. Math. 5: 243

Das A 1991 Analysis of spiraling vortical flows around slender delta wings moving in an inviscid medium. Z. Angew. Math. Mech. 71: 465-471

Das A, Longo J M A 1994a Numerical computation of vortical flow fields of double-delta wings moving in a compressible medium. Z. Angew. Math. Mech. 74: 475-486

Das A, Longo J M A 1994b Numerical analysis of the vortical flow field around a delta-shaped wing-canard configuration moving in a compressible medium (to be published in $A I A A J$.)

Deese J E, Agarwal R K 1987 Navier-Stokes calculations of transonic viscous flow about a wing body configuration. AIAA paper 87-1200

Deese J E, Agarwal R K 1988 Euler/Navier-Stokes calculations of transonic flow past fixed-and rotary-wing aircraft configurations. Transonic Symposium Proceedings vol. 1, part 2, NASA-LARC, Hampton, pp. 521-545 
Degani D, Schiff L B 1983 Computation of supersonic viscous flows around pointed bodies at large incidence. AIAA paper 83-0034

Drougge $G 1988$ The international vortex flow experiment for computer code validation. ICAS-Proceedings 1988 , vol. 1, pp. 25-41

Eberle A 1987 Characteristic flux averaging approach to the solution of Euler's equation. VKI Lecture Series 1987-04

Elsenaar A, Hoijemakers H W M 1990 An experimental study of the flow over a sharp-edged delta wing at subsonic and transonic speeds. AGARD FDP Symposium on Vortex Flow Aerodynamics. AGARD-CP-494, pp. 15.1-15.19

Eriksson L E 1982 Generation of boundary-conforming grids around wing-body configurations using transfinite interpolation. $A I A A J$. 20: 1313-1320

Eriksson L, Rizzi A 1983 Computation of vortex flow around a canard-delta wing combination. Proc. of 5th GAMM Conference on Numerical Methods in Fluid Mechanics pp. 65-80

Esch H 1989 Force measurements on four 1:100 HERMES shape-derivatives at subsonic and supersonic Mach numbers. DLR-IB 39113-89-C-21, 1989

Findling A, Herrmann U 1991 Development of an efficient grid generation. Proc. 3rd Int. Conf. on Numerical Grid Generation in Comput. Fluid Dynamics and Related Fields. Barcelona, Spain, pp. 781-792

Goddard J L, Jacquotte O P, Gisquet D 1991 Analyse detaillee de 1'interaction voilure-nacelle d'un avion de transport civil. AGARD Symposium on Aerodynamic Engine/Airframe Integration, Texas

Hartwich P M, Hsu C H, Luckring J M, Liu C H 1988 Aerodynamic application of an efficient incompressible Navier-Stokes solver. ICAS Proc. vol. 2, pp 1417-1427

Hilgenstock A 1990 Ein Beitrag zur numerischen Simulation der Transsonischen Strömung um einen Deltaflügel durch Lösung der Navier-Stokes'schen Bewegungsgleichungen. DLR-FB 90-13

Hilgenstock A, Vollmers H 1990 On the simulation of compressible turbulent flows past delta wings, delta wing-body and delta wing-canard. $A G A R D$ FDP Symposium on Vortex Flow Aerodynamics, AGARD-CP-494, pp. 7.1-7.14

Hummel D 1988 Documentation of separated flows for computational fluid dynamics validation. AGARD-CP-437, vol. 2, pp. 15.1-15.24

Jameson A 1985 Multigrid algorithms for compressible flow calculations. MAE Report 1743, Princeton University

Jameson A, Schmidt W, Turkel E 1981 Numerical simulation of the Euler equations by finite volume method using Runge-Kutta time stepping schemes. AIAA paper 81-1259

Johnson D A, King L S 1984 A new turbulence closure model for boundary layer flows with strong adverse pressure gradients and separation. AIAA paper 84-0175

Krause E, Liu C H 1989 Numerical studies of incompressible flow around delta and double-delta wings. Z. Flugwiss. 13: 291-301

Kroll N 1989 Berechnung von Strömungsfeldern um Propeller und Rotoren im Schwebeflug durch die Lösung der Euler-Gleichungen. Dissertation Technische Universitat Braunschweig, DLR-FB 89-37

Kroll N, Gaitonde D, Aftosmis M 1991 A systematic comparative study of several high resolution schemes for complex problems in high speed flows. AIAA paper 91-0636

Kroll N, Jain R 1987 Solution of two-dimensional Euler equations - experience with a finite volume code. DFVLR-FB 87-41

Kroll N, Radespiel R, Rossow C -C 1989 Experiences with explicit time-stepping schemes for supersonic flow fields. 8th GAMM Conf. on Numerical Methods in Fluid Dynamics (Delft: Vieweg)

Kroll N, Rossow C -C 1990 A high resolution cell vertex TVD scheme for the solution of the two- and three-dimensional Euler equations. 12th Int. Canf. on Num. Methods in fluid dynamics. Lecture Notes in Physics 371:

Kumar A, Das A 1988 Computation of vortex flow on a delta wing at transonic speed. IUTAM Symposium Transsonicum III pp. 317-328

Lambourne N C 1982 Compendium of unsteady aerodynamics measurements, AGARD-R-702

Lax P D, Wendroff B 1966 System of conservation laws. Commun. Pure Appl. Math. 13: 217-237

Lin C Q, Pahlke K 1991 Numerical solution of Euler equations for airfoils in arbitrary unsteady motion. DLR-FB 129-91/17 
Longo J M A 1988 Research on three different Euler schemes applied to a delta wing with vortical flows. DGLR STAB Symposium

Longo J M A 1992a Untersuchungen der Umströmung von Deltaflügel-Konfigurationen mit und ohne Canard durch numerische Lösung der Euler-Gleichungen. Dissertation Technische Universitat Braunschweig

Longo J M A 1992b Viscous transonic flow simulation around a transport aircraft configuration. DGLR Jahrestagung

Longo J M A, Das A 1990 Numerical simulation of vortical flows over close-coupled canard-wing configuration. AIAA 90-3003

MacCormack $\mathrm{R} 1982$ A numerical method for solving the equations of compressible viscous flow. $A I A A$ J. 20: 1275-1281

MacCormack R 1985 Current status of numerical solutions of the Navier-Stokes equations. AIAA paper $85-0032$

Maynard J D, Murphy M P 1950 Pressure distributions on the blade sections of the NACA 10(3) (066)-033 propeller under operating conditions. NACA RM L9L12

Murman E M, Rizzi A 1986 Application of Euler equaitona to sharp edge delta wing with leading edge vortices. AGARD Conf. Proc. No. 412 pp. 15-1 to 15-13

Newsome R W, Kandil O A 1987 Vortical flow aerodynamics - Physical aspects and numerical simulation. AIAA paper $87-0205$

Nixon D (ed.) 1989 Unsteady transonic aerodynamics. AIAA, Progress in Astronautics and Aeronautics, vol. 120

Oelker H -Chr 1990 Aerodynamische Untersuchungen an kurzgekoppelten Entenkonfigurationen bei symmetrischer Anströmung. Dissertation Technische Universitat Braunschweig, ZLR Forsc. Bericht der Technische Universitat Braunschweig 90-01

Pahlke K, Kroll N 1991 Grid generation for multi-blades and calculations with a 3-D Euler-code for helicopter rotors in hover. DLR-IB 129-91/4

Pan D, Lomax H 1986 A new approximate LU factorization scheme for the Reynold's-averaged Navier-Stokes equations. AIAA paper $86-0337$

Pfitzner M, Weiland C 1987 Three-dimensional Euler solution for hypersonic Mach numbers. AGARD-CP 429

Pulliam T H, Steger J L 1980 Implicit finite difference simulation of three-dimensional compressible flow. AIAA J. 18: 159-167

Radespiel R 1988 Grid generation around transport aircraft configurations using a multiblock structured computational domain. In AGARDOgraph No. 309 pp. 139-153

Radespiel R 1989 A cell-vertex multigrid method for the Navier-Stokes equations. NASA-TM 101557

Radespiel R, Kroll N 1985 Progress in the development of an efficient finite volume code for the three dimensional Euler equations. DFVLR-FB 85-31

Radespiel R, Kroll N 1990 A multigrid scheme with semicoarsening for accurate computation of viscous flows. 12th Int. Conf. on Numerical Methods in Fluid Dynamics. Lecture Notes in Physics. 371:

Radespiel R, Quast A 1989 Kraftmessungen, Druckverteilungen und Strömungssichtbarmachung am Raumgleitermodell FALKE im Niedergeschwindigkeitsbereich. DLR IB-129-89/37

Radespiel R, Rossow C -C, Swanson R C 1990 An efficient cell-vertex multigrid scheme for the three-dimensional Navier-Stokes equations. $A I A A J .28$ : 1464-1472

Radespiel R, Swanson R C 1991 Progress with multigrid schemes for hypersonic flow problems. NASA CR 189579 ICASE Report No. 91-89

Raj P, Sikora J S, Keen J M 1988 Free vortex flow simulation using a three-dimensional Euler aerodynamics method. ICAS Proc. vol. 1, pp. 604-617

Redeker G, Müller R, Ashill P R, Elsenaar A, Schmitt V 1987 Experiments on the DFVLR-F4 wing-body configuration in several European wind tunnels. AGARD-CP 429, pp. 2.1-2.15

Rizetta D P, Shang J S 1987 Numerical simulation of leading edge vortex flows. AIAA J. vol. 24, pp. 237-245

Rizk Y M, Chaussee D S, McRae D S 1981 Computation of hypersonic flows around threedimensional bodies at high angles of attack. AIAA paper 81-1261

Roe P L 1981 Approximate Riemann solvers, parameter vectors and difference schemes. $J$. Comput. Phys. 43: 357-372

Rossow C -C 1987 Comparison of cell centered and cell vertex finite volume schemes. In Proc. 7th GAMM Conference, Louvain-La-Neuve/Belgium 
Rossow C -C 1989 Berechnung von Strömungsfeldern durch Lösung der Euler-Gleichungen mit einer erweiterten Finite-Volumen Diskretisierungsmethode. Dissertation der Techniche Universitat Braunschweig, DLR-FB 89-38

Rossow C -C 1991a Flux balance splitting: A second order accurate cell vertex upwind scheme. 9th GAMM Conference on Numerical Methods in Fluid Mechanics, Lausanne/Schweiz

Rossow C -C 1991b Efficient computation of inviscid flow fields around complex configurations using a multiblock multigrid method. 5th Copper Mountain Conf. of Multigrid Methods

Rossow C -C, Goodard J -L, Hoheisel H, Schmidt V 1992 Investigation of propulsion integration interference effects on a transport aircraft configuration. 28th Joint Propulsion Conference, Nashville, TN, AIAA paper 92-3097

Rossow C -C, Ronzheimer A 1991a Investigation of interference phenomena of modern wingmounted high-bypass-ratio engines by the solution of the Euler-equations. $A G A R D$ Symposium on Engine/Airframe Integration, AGARD-CP-98, pp. 5.1-5.11

Rossow C -C, Ronzheimer A 1991b Multiblock grid generation around wing-body-enginepylon configurations. Numerical Grid Generation in Computational Fluid Dynamics and Related Fields pp. 357-368

Rudnik R 1991 Erweiterung eines dreidimensionalen Euler-Verfahrens zur Berechnung des Strömungsfeldes um Nebenstromtriebwerke mit Fan- und Kernstrahl. Z. Flugwiss. 15(5):

Scherr S, Das A 1988 Basic analysis of the flow fields of slender wings using the Euler equations. ICAS Proc. vol. 2, pp. 1428-1436

Schmitt V, Charpin F 1979 Pressure distribution on the ONERA M6 wing at transonic Mach numbers. AGARD-AR-138

Schöne J, Bidault J 1991 Calculation of inviscid flow around a reentry configuration at supersonic speed. AIAA paper 91-0391

Schöne J, Streit Th, Kroll N 1990 Aerodynamische Berechnungen für eine HERMES-Studienkonfiguration bei Überschallanströmung durch Lösung der Euler Gleichungen. DGLRJahrestagung 1990, 1.-4. Oktober 1990, Friedrichshafen. Jahrbuch 1990 der DGLR

Schöne J, Streit Th, Kroll N 1991 Steps towards an efficient and accurate method solving the Euler equations around a re-entry configuration at super- and hypersonic speed. Proceedings 1st European Symposium on Aerothermodynamics for Space Vehicles, Noordwijk

Schwarz W 1986 Elliptic grid generation system for three-dimensional configurations using Poisson's equation. Proc. Ist Int. Conf. on Numerical Grid Generation in Comput. Fluid Mech., (Pineridge Press) pp. 341-352

Sonar Th 1989 Grid generation using elliptic differential equations. DFVLR-FB 89-15

Sonar Th, Radespiel R 1986 Geometric modelling of complex aerodynamic surfaces and threedimensional grid generation. In: "Numerical Grid Generation in CFD", Proc. 2nd Int. Conf. Miami (Pineridge Press)

Steger J, Warming R F 1976 Flux vector splitting of inviscid gasdynamic equations with application to finite difference methods. NASA TM-78605

Swanson R C, Radespiel R 1991 Cell centered and cell vertex multigrid schemes for the Navier-Stokes equations. $A I A A J$. 29: 697-703

Thompson J F, Warsi Z U H, Mastin C W 1985 Numerical grid generation. Foundation and applications (Amsterdam: North Holland)

Van Leer B 1985 Upwind difference methods for aerodynamic problems governed by the Euler equations. Lect. Appl. Math. 23: 327-336

Van Leer B, Thomas J L, Roe P L, Newsome R W 1987 A comparison of numerical flux formulas for the Euler and Navier-Stokes equations. AIAA paper 87-1104

Volpe G, Jameson A 1988 An efficient method for computing transonic and supersonic flows about aircrafts. ICAS-88-4.8.3, pp. 1224-1235

Whitfield D L, Janus D M 1984 Three-dimensional unsteady Euler equations solution using flux vector splitting. AIAA paper $84-1552$

Whitfield D L et al 1987 Three-dimensional unsteady Euler solutions for propfans and counterrotating propfans in transonic flow. AIAA paper 87-1197

Wichmann G 1992 Untersuchungen zur Flügel-Rumpf-Interferenz durch Anwendung eines Eulerverfahrens für kompressible Strömungen. Dissert, Technische Universitat Braunschweig 